\title{
Evolution in students' understanding of thermal physics with increasing complexity
}

\author{
Elon Langbeheim \\ Department of Science Teaching and the Department of Materials and Interfaces, \\ Weizmann Institute of Science, Rehovot, Israel, 76100
}

Samuel A. Safran

Department of Materials and Interfaces, Weizmann Institute of Science, Rehovot, Israel, 76100

Shelly Livne and Edit Yerushalmi

Department of Science Teaching, Weizmann Institute of Science, Rehovot, Israel, 76100

(Received 25 June 2012; published 12 November 2013)

\begin{abstract}
We analyze the development in students' understanding of fundamental principles in the context of learning a current interdisciplinary research topic — soft matter — that was adapted to the level of high school students. The topic was introduced in a program for interested 11th grade high school students majoring in chemistry and/or physics, in an off-school setting. Soft matter was presented in a gradual increase in the degree of complexity of the phenomena as well as in the level of the quantitative analysis. We describe the evolution in students' use of fundamental thermodynamics principles to reason about phase separation - a phenomenon that is ubiquitous in soft matter. In particular, we examine the impact of the use of free energy analysis, a common approach in soft matter, on the understanding of the fundamental principles of thermodynamics. The study used diagnostic questions and classroom observations to gauge the student's learning. In order to gain insight on the aspects that shape the understanding of the basic principles, we focus on the responses and explanations of two case-study students who represent two trends of evolution in conceptual understanding in the group. We analyze changes in the two case studies' management of conceptual resources used in their analysis of phase separation, and suggest how their prior knowledge and epistemological framing (a combination of their personal tendencies and their prior exposure to different learning styles) affect their conceptual evolution. Finally, we propose strategies to improve the instruction of these concepts.
\end{abstract}

DOI: 10.1103/PhysRevSTPER.9.020117

PACS numbers: 01.40.gb

\section{INTRODUCTION}

The increasing importance of interdisciplinary topics and approaches in current scientific and technological research stands in contrast to the compartmentalized manner in which introductory science courses are commonly taught. In fact, many students who complete introductory physics fail to view even physics itself as a coherent knowledge structure [1]. This failure suggests that after completing an introductory physics course students are almost certainly unable to meet the challenge [2] of relating the physics that they studied to phenomena encountered in other fields such as chemistry and biology. Furthermore, they are probably not able to approach, simplify, and quantify the complex systems characteristics of these fields with the tools offered by physics. On the other hand, cross-disciplinary epistemological boundaries often prevent biology and chemistry students from intellectual engagement in introductory physics courses.

Published by the American Physical Society under the terms of the Creative Commons Attribution 3.0 License. Further distribution of this work must maintain attribution to the author(s) and the published article's title, journal citation, and DOI.
Statistical thermodynamics is a framework that unifies the behavior of many systems of interest to interdisciplinary research in physics, chemistry, and biology. Its presentation in introductory courses is typically limited to ideal gas systems with no interparticle interactions [3]. This limited scope prevents students from understanding and appreciating the wide variety of fascinating, emergent phenomena in interacting molecular systems in physics, chemistry, and biology [4].

To substantially broaden the scope of the traditional thermal physics unit in introductory courses, we proposed a novel interdisciplinary curriculum on soft matter [5]. The curriculum was introduced in a program for interested Israeli high school students majoring in chemistry and/or physics [6]. Soft matter is an interdisciplinary field that uses statistical thermodynamics to study the behavior of materials such as fluid mixtures, colloidal dispersions, polymers, and surfactant solutions; these are important in both chemical technology and as models of biological systems such as the cell membrane. Soft matter is an appropriate choice for an introductory level interdisciplinary course because the central methods used to teach the theory can be presented in a manner suited to the mathematical and scientific background of high school students. Our soft matter 
program establishes its intellectual basis with a presentation of introductory level thermal physics using a microscopic, statistical perspective, as proposed by several physics educators $[7,8]$. The complexity of the phenomena presented in the program gradually evolves from traditional, ideal systems to systems with interparticle interactions.

Several other studies have reported how advanced science topics (e.g., quantum physics, astrophysics) can be introduced to capable and interested high school students $[9,10]$. Similar to the soft matter program, these courses include sophisticated analyses and authentic experiences intended to stimulate the curiosity of students with relatively little background in science. Because of the considerable training and expertise required to analyze advanced topics, educators commonly have no alternative but to "popularize" these topics, possibly obscuring the manner in which the basic principles of science play out in these systems. The above-mentioned programs take an intermediate approach, between popularization and rigorous presentation of a topic that includes intensive practice of problem solving. The intermediate approach engages the students in the intellectually challenging details of the modeling of systems using basic principles but still does not focus on the intensive practice needed to bring students to competence as problem solvers in the field.

The learning in such programs that introduce advanced topics to relatively young students has been studied from several aspects such as students' understanding of models, development of inquiry skills, and attitudes towards learning science [9]. However, one might question the impact of these approaches on students' conceptual understanding of the fundamental physics principles presented in these courses. In particular, does the complexity of the subject matter obscure the fundamental scientific principles that underlie these constructs, resulting in students deemphasizing or even forgetting the fundamentals? In the context of our course we have studied the evolution of high school students' conceptualization of fundamental principles of thermal physics as the level of difficulty and the soft matter systems treated increased in complexity.

Phase separation of binary mixtures is a central phenomenon in soft matter and its analysis in terms of fundamental thermodynamics principles forms the basis for the modeling of other phenomena such as the self-assembly of amphiphilic (e.g., soap, lipid) molecules. At first glance, phase separation (that results in a decrease in the system entropy [11]) can seem to contradict the second law of thermodynamics. Naively, the second law of thermodynamics mandates that isolated systems composed of two different types of noninteracting particles that are initially segregated should always mix so that the entropy of the combined system will increase. However, in many interacting systems that can exchange heat with a thermal reservoir, the opposite can also occur, and an initially mixed system can phase separate (depending on the temperature). Typically, one does not measure a temperature change in the reservoir, since the system size (and hence the energy it dissipates to the reservoir) is negligible relative to the size of the reservoir. Thus, phase separation can (incorrectly) be perceived as a violation of the second law of thermodynamics if we focus only on the system's entropy (which indeed decreases).

The standard theoretical analyses of phase separation are predicated on the minimization of the system free energy. For a system in thermal equilibrium with a much larger reservoir, minimization of the free energy is equivalent to the maximization of the total (system plus reservoir) entropy as mandated by the second law of thermodynamics. Internalizing this connection and not just using it as a technical tool is required to reconcile phase separation with the second law. However, it is possible that the elaborate procedures required for the application of free energy to specific systems may obscure the fundamental connection of the free energy and the second law. Indeed, misinterpreting processes as contradicting the second law of thermodynamics also occurs in the analysis of chemical reactions in which products are more ordered than the reactants [12] and in biology when considering processes such as photosynthesis in which organized structures are formed [13].

This paper analyzes the evolution of student understanding of fundamental concepts of thermal physics (such as entropy, internal energy, and the second law of thermodynamics) in the context of phase separation of mixtures as the complexity of their presentation increases. Thereafter, it suggests possible explanations for the results in terms of students' epistemologies that are prone to enable or impede their conceptual evolution. We do so by revisiting the findings, interpreting them in terms of students' epistemological framing [14] and their role in shaping various paths of conceptual evolution that were found. In particular, we suggest how students' disciplinary backgrounds (i.e., physics or chemistry majors) shape both their conceptual resources and epistemological framing. In the following sections we first survey prior research related to student understanding of the concepts of thermal physics in the context of traditional introductory chemistry and physics curricula that can serve as a baseline for our study and then outline our methodology and findings.

\section{LITERATURE REVIEW}

The systems studied in soft matter are at the boundary between chemically bonded systems usually discussed in introductory chemistry and ideal gases that are usually discussed in introductory physics, since the interactions between particles do exist but are much weaker than chemical bonds. In addition, the target audience of the soft matter program consisted of students majoring in high school physics or high school chemistry. Thus, to 
discuss student difficulties related to soft matter one should consider the relevant science education literature in both chemistry and physics. This literature includes theoretical discussions of the proper definitions of essential scientific concepts that are needed for the statistical-thermodynamic analysis of systems of soft matter (such as internal energy, entropy, free energy, and the guiding principles of the laws of thermodynamics), the preferred logical flow of their presentation, and empirical studies concerning student difficulties. We note that most of the empirical studies do not use the resources and epistemology constructs, and some even use "misconceptions" rhetoric. However, their findings are important as an initial perspective on student difficulties that will later shed light on the analysis of the management of thermodynamics resources in the soft matter program. In the following, we summarize a few of these papers.

\section{A. Studies concerning internal energy and the first law of thermodynamics}

The standard presentation of basic concepts related to energy varies significantly between chemistry and physics in both introductory courses and their associated textbooks. For example, physics curricula commonly define energy changes of isolated bodies, using the work-energy theorem derived from Newton's second law, where potential energy is defined as the work performed by conservative forces acting upon an object. In contrast, in most chemistry curricula and in thermodynamics courses, energy is discussed in the context of multiparticle systems and potential energy is assigned to chemical bonds between two particles. To remedy this discrepancy, several physics educators [15] propose an approach to energy instruction based on the first law of thermodynamics. The first-law approach relates the change in the internal energy of a system (the statistical average of the sum of the potential and kinetic energies) to the external work done on the system and to heat transfer to or from it. External work is induced by forces external to the system, and is distinguished from work done by conservative forces that arise from the interparticle interactions within the system that determine the change in the overall potential energy.

The understanding of energy in the context of multiparticle systems poses several difficulties related to the distinction between various ideas and to the different approaches used in the analysis of energy changes. Energy-related concepts such as temperature and heat are not easily distinguished by high school students [16]. In a study performed in introductory physics courses, about half of the student sample incorrectly stated that no heat transfer occurred during the compression of a gas when the initial and final temperatures of the gas are the same [17], thus confusing heat transfer (which indeed occurs in the process) and the change in temperature (which remains constant). Similar findings were reported regarding students who completed an undergraduate course in chemistry [18]. Moreover, many of the students in an algebrabased introductory physics courses could not discern the terms "heat" and "internal energy" [19].

In addition to the difficulty of distinguishing between similar concepts, the proper approaches to analyze energy changes are also not easily applied. For example, only $10 \%$ of the students in the study mentioned above [19] could explain that the temperature of an ideal gas increases upon compression, because work is performed on the gas. Instead, students invoked the ideal gas law $(P V=n R T)$ which was insufficient to properly solve the problem since no information was given regarding the pressure of the gas. A second study [20] showed that the use of the first law of thermodynamics is not invoked by introductory physics students studying situations in which the internal structure of a system cannot be ignored when applying the concepts of work and energy. In this context, students tend to analyze the change in the mechanical energy of a system based on changes in its potential and kinetic energies. They do so even when the information provided regarding the magnitudes of these changes does not enable a reliable prediction of these quantities. In this example, an analysis in terms of the work done by external forces (first law of thermodynamics) would have led to a reliable prediction regarding the change in the energy of the system.

The distinction between the system and surroundings and the analysis of their interplay is an especially difficult feature of energy analysis. In a physics context, a recent paper reported that students recognize that two objects (e.g., a block and spring) can be grouped together as a system [21]. In the chemistry context of an exothermic reaction that takes place in solution, students thought that heat was transferred from the reactants to the products when in fact heat was transferred from the system (i.e., the reactants and the products of the chemical reaction) to the surroundings (i.e., the solvent) [22].

\section{B. Studies concerning entropy and the second law of thermodynamics}

Many authors advocate a coherent presentation of entropy as the number of ways that energy can be distributed or spread among the particles and energy levels in the system [23] while others prefer to present it in a more general manner as a measure of "knowledge" or "information" about the locations (or energies) of the particles in the system [24]. Both of these approaches support presenting entropy using an atomistic, statistical approach [7]. The statistical definition of entropy is presented through spatial counting of microstates of particles that are distributed in a particular volume and the distributions of energy quanta between these particles [25]. Some textbooks [26] and even Boltzmann [27] himself used the term "disorder" to explain entropy; however, presenting entropy as disorder 
may lead students to overgeneralize the use of the term to describe processes in macroscopic objects (e.g., misuse of equilibrium entropy in a "driven" process such as the shuffling of cards) that do not occur through thermal activation [28]. In addition, students may identify images of systems that seem more disorganized as having greater entropy, instead of relating it more specifically to a greater number of possible configurations.

Several empirical studies have documented students' conceptual difficulties related to entropy and the second law of thermodynamics in both introductory physics courses and chemistry courses. One report observed that it was difficult for university chemistry students to adopt the scientific definition for entropy [29] presented in the course and referred instead to nave ideas of "randomness" and "disorder" [30]. A different study, focused on thermal equilibration [31], found that after taking an introductory physics course, many students think that the total entropy is conserved in spontaneous processes. This may indicate that entropy is conceptualized as a "form of energy" rather than a measure of "spreading" or a characterization of energy or spatial distributions [32].

In introductory physics courses, the understanding of the second law of thermodynamics was also studied in the context of heat engines [33]. In the analysis of cyclic processes in heat engines, most students invoked only the first law of thermodynamics which seemed relevant to the problem, but disregarded the second law of thermodynamics that determines whether the device functions at its ideal efficiency [34]. In the context of chemical reactions many students incorrectly stated that the entropy of the system must always increase, irrespective of changes in the entropy of the surroundings [18].

Another important thermodynamic concept is the free energy. One study found that introductory chemistry students tended to confuse free energy with enthalpy or with an energy that can be extracted in the reaction [35]. Instead of using the free energy to determine if a process was spontaneous or not, students indicated that the decrease in enthalpy of the system was the strongest determinant of spontaneity and that processes that are not exothermic cannot be spontaneous [36].

To conclude, the empirical studies mentioned here indicate many conceptual challenges related to a proper understanding of internal energy, entropy, free energy, and the second law of thermodynamics, all of which are necessary to understand phenomena in soft matter systems. Among these challenges are difficulties in choosing a proper approach to predict the change in the internal energy of systems, an inability to define entropy in terms of the distribution of energy among the particles of the system, conceptualizing entropy as a "form of energy" that must be conserved, focusing on the entropy of the system and disregarding the environment, and confusing free energy with internal energy. Many of these difficulties were expressed by students in the soft matter course, and we touch upon these points in our analysis and discussion.

\section{METHODOLOGY}

We now describe the methods we used to assess students' knowledge (conceptual) resources and their management in the analysis of phase separating systems in which the system entropy decreases. We probed the evolution of the knowledge resources and their management using questionnaires containing conceptual problems at three, increasingly complex stages of the curriculum. In the following, we first describe the stages of the curriculum, the gradual increase in their complexity, and the core ideas they convey. Next, we outline the profile of the participants by comparing their performance on diagnostic problems to that of introductory college students as observed in other studies. We then explain the choice of the two case studies we focus on: a chemistry major and a physics major, whose answers to the diagnostic problems are used to describe the evolution of their knowledge resources and management when solving a problem on phase separation. Case-study research has been used to investigate learning in either unique or conventional curricula [37]. Next, we describe the data collection tools and finally our analysis methods.

\section{A. Curriculum description}

The core of the soft matter course focuses on a quantitative analysis of the equilibrium properties of soft matter that is based on microscopic statistical models (e.g., lattice models, random walk models). These topics are usually taught at the advanced undergraduate or graduate levels. Therefore, several mathematical simplifications and scientific adaptations were incorporated in order to make the presentation appropriate for high school students. However, the fact that models of soft matter do not rely on quantum mechanics, sophisticated electromagnetism, or complex mechanics means that these simplifications still permit both the qualitative and quantitative explanations (based on simple statistical and thermodynamic ideas) to shine through. The curriculum development was accompanied with formative evaluation of three, two-year cycles of the program, each involved about 15 students. Analysis of students' worksheets, video documentation of the lessons, diagnostic and feedback questionnaires, as well as reflective documentation from the teachers served to refine and elaborate the curriculum.

The study was conducted in a two-year program offered to 11th grade students at an off-school, regional science center located in a university setting. The program was designed in the spirit of a "spiral curriculum" in which the fundamental concepts were initially presented only briefly and later on were reintroduced in greater detail, depth, and sophistication. The curriculum is roughly divided into four stages. Stage A, which spans six 2.5 hour meetings [38], 
encompasses a qualitative, descriptive analysis of ideal gas expansion and phase separation in a colloidal suspension in terms of a microscopic, statistical representation of the principles of thermodynamics. Stage B spans four meetings and incorporates a rigorous, quantitative analysis of ideal systems in terms of the concepts and principles introduced in stage A. For example, the definition of the entropy in terms of both spatial configurations and energy distributions was discussed. This stage culminates with the introduction of the free energy, which is shown to be equivalent to the second law of thermodynamics for a system in thermal contact with a heat bath. The utility of the free energy in allowing one to focus on the internal energy and entropy of the system, where the heat bath serves only to fix the temperature in equilibrium, is examined. Stage C spans eight meetings and focuses on a modeling sequence [5] that involves the application of free energy minimization to systems of interacting particles. The free energy of attracting particles that show transitions from mixed to phase separated states is analyzed quantitatively in terms of the internal energy and entropy using a mean-field approximation. This approach is further used to examine specific soft matter systems in which interparticle interactions are important, such as wetting or capillarity, self-assembly, and polymers. A brief description of the topics studied in each stage of the curriculum is given in Table II and in the Appendix.

In stage A students explored intriguing phenomena intended to create a need for a more accurate scientific language that can be used to explain these phenomena. Once the scientific language was introduced, students were asked to employ it to describe in a qualitative manner various experimental phenomena. In this stage as well as in stage $\mathrm{B}$, students refined their understanding of the scientific concepts and principles in the context of simple conceptual questions. They were asked to explain and defend their ideas, first in groups of two or three students and later in class discussion. With the increase in the complexity of the mathematical derivations involved in stage $\mathrm{C}$, the type of tasks assigned shifted towards those in which students were asked to analyze an expertconstructed model of the phenomena. This analysis could include translating between various representations of the model (observations and measurements, equations, simplified visualizations, and graphs) or restructuring the modeling sequence (for example, via concepts maps). This stage was accompanied by troubleshooting tasks intended to allow students to compare both scientifically acceptable as well as nonadequate applications of the scientific concepts and principles used in the modeling sequence. In stage D, students conducted independent experimental or computational inquiry projects that were then analyzed using the theoretical modeling of soft matter phenomena with the approach of free energy minimization. They wrote a final paper explaining both the experimental methods applied as well as the relation between observation and theory. Peer evaluation accompanied this stage as a means of supporting the students in coping with the challenge of presenting the final paper. The performance of students in stage $\mathrm{D}$ is not part of this study. A more detailed discussion of the curriculum, including the mathematical adjustments and supporting teaching activities that were used, can be found in a separate paper [5].

\section{B. Participants \\ 1. Group characteristics}

Fourteen students joined the program after hearing about it from their science teachers, from a school presentation by a representative of the program, or through the internet. Seven majored in high school chemistry, four in physics, and three in both. The group consisted of eight female and six male students. The students joined the program voluntarily and were not screened via an entrance exam. We estimate the level of the group using two measures. First, students accepted to the program were required to pass their school science courses with a minimum grade of $85 / 100$. Second, the group's performance on a diagnostic problem on thermal equilibration was compared to that of college students taking a traditional introductory college physics course [31]. We compared the groups' performance before and after stage B in which entropy and the second law of thermodynamics were presented using a microscopic statistical model. The pre-stage B question was administered in the first questionnaire after a descriptive presentation of the basic concepts, in which the second law of thermodynamics was introduced as the tendency for the disorder of the Universe to increase, and heat was introduced as the flow of thermal energy from objects of high temperature to objects of low temperature. The level at which these concepts were presented was similar to that of the physical science school course content in the U.S. according to the National Science Education Standards [39] (the term "entropy" is not mentioned in this document, only the tendency of matter to "get less ordered"). This allowed a comparison of the preinstruction results of the group of high school students studying soft matter with that of a group of college students. The post-stage B questionnaire (administered after stage B that treated the concepts of statistical thermodynamics in the context of ideal systems) allowed us to compare the learning of similar topics (the college students received a researchbased tutorial session). Table I shows the diagnostic problem and the comparison of the percentage of correct responses given by the soft matter students to those given by college students.

We found no significant difference $(p<0.05)$ between the answers of the two groups, both pre- and postinstruction, using a binomial proportions test [40]. This result indicated that the level of the two groups is comparable; the understanding of second law of thermodynamics in the 
TABLE I. A comparison of student correct answers to the thermal equilibration problem, expected answers are in bold.

\begin{tabular}{|c|c|c|c|c|}
\hline $\begin{array}{l}\text { An object is placed in a thermally isolated room } \\
\text { that contains air. The object and the air in the room } \\
\text { are initially at different temperatures. The object } \\
\text { and the air in the room are to exchange energy } \\
\text { with each other. }\end{array}$ & $\begin{array}{l}\text { Soft matter } \\
\text { students }(N=14) \\
\text { first questionnaire } \\
\text { "pre" }\end{array}$ & $\begin{array}{l}\text { College- } \\
\text { Preinstruction } \\
(N=191)\end{array}$ & $\begin{array}{l}\text { Soft matter students } \\
(N=11) \text { second } \\
\text { questionnaire } \\
\text { "post" }\end{array}$ & $\begin{array}{l}\text { College- } \\
\text { Postinstruction } \\
\quad(N=91)\end{array}$ \\
\hline $\begin{array}{l}\text { During the process does the entropy (disorder) } \\
\text { of the object increase, decrease, remain the } \\
\text { same, or is this not determinable with the } \\
\text { given information? }\end{array}$ & $61.5 \%$ & $53 \%$ & $82 \%$ & $73 \%$ \\
\hline $\begin{array}{l}\text { During the process does the entropy (disorder) of } \\
\text { the air in the room increase, decrease, remain } \\
\text { the same, or is this not determinable with the } \\
\text { given information? }\end{array}$ & $50 \%$ & $52 \%$ & $82 \%$ & $73 \%$ \\
\hline $\begin{array}{l}\text { During the process does the entropy (disorder) of } \\
\text { the object plus air in the room increase, } \\
\text { decrease, remain the same, or is this not } \\
\text { determinable with the given information? }\end{array}$ & $15.4 \%$ & $21 \%$ & $73 \%$ & $69 \%$ \\
\hline
\end{tabular}

context of thermal equilibration of the soft matter students after stage A was similar to the general undergraduate student population who acquired it in their high school science education. In addition, after stage B we saw a similar increase in the number of correct responses of the high school and the introductory college groups.

In addition to the comparison of student responses to the thermal equilibration problem, we compared the responses of the high school soft matter students to those of college students to a problem involving the expansion of an ideal gas as shown in the Appendix. Here again, we saw no significant difference in the performance of the two populations before and after instruction. However, due to the small sample size of the college students that participated in that study, our conclusions regarding this comparison are less rigorous compared with those detailed in Table I.

\section{Choosing the case studies}

Case studies should portray an authentic pathway of learning in the course; that is, they should represent a typical performance, should have a substantial exposure to the instruction, and should have made productive contributions to the data [41]. In order to select the students based on typical performance, we first reviewed the responses of the entire group and identified two trends of conceptual evolution as the program continued: One subgroup of students were gradually able to adequately manage the conceptual resources in a manner that was aligned with the scientific interpretation, while the other subgroup did not. We decided to choose one student from each subgroup as representatives of these two different learning trajectories in order to convey a balanced presentation of the learning that took place in the program. Ruben and Simon satisfied these criteria and were thus chosen as representatives of each subgroup. They were among those that attended most of the meetings, completed the worksheets, and participated in class discussions. Ruben majored in chemistry and studied advanced high school math and chemistry in parallel with the soft matter program. Simon is a physics major who also took classes in undergraduate mathematics at the Open University in parallel with the soft matter program.

\section{Data collection}

To maintain ecological validity we utilized two primary data collection tools that are based on authentic classroom data observations and materials produced by the students as they participated in the program. The first was a set of three open-ended diagnostic questionnaires that were administered after each stage of instruction. The second data collection tool used was video documentation of class discussions. Table II shows the time line of data collection within the structure of the curriculum.

The diagnostic questionnaires shared several isomorphic questions involving the application of the laws of statistical thermodynamics in analyzing the phenomena of phase separation. In this study we focus on a phase separation scenario, shown in Fig. 1. The other problems [42] were used for triangulation of the analysis of the responses to the phase separation problem. In the transcripts of classroom discussions we focused on episodes in which the ideas raised in the diagnostic questionnaire were further discussed by the students.

The generic format of the phase separation problem was the following: A process in which a system phase separates was introduced, in which the temperature of the system at the beginning and at the end of the process was the same. Based on this information, the students were asked to 
TABLE II. Teaching sequence outline and corresponding data collection points.

\begin{tabular}{|c|c|c|}
\hline & Main curricular messages in stage & Data \\
\hline $\begin{array}{l}\text { Stage A-Descriptive } \\
\text { treatment of } \\
\text { principles of } \\
\text { statistical } \\
\text { thermodynamics }\end{array}$ & $\begin{array}{l}\text { Meetings } 1 \text { and 2: Demonstrations of unusual materials, explanation of a } \\
\text { macrostate via microscopic properties. } \\
\text { Meetings } 3 \text { and 4: Statistical concepts for linking the microscopic picture with } \\
\text { the macroscopic properties: probability, mean, disorder. Second law of } \\
\text { thermodynamics defined as the tendency of the universe to evolve towards } \\
\text { a state of maximal disorder. } \\
\text { Meetings } 5 \text { and 6: Internal energy is the sum of the average kinetic energy and } \\
\text { potential energy (interactions) of a multiparticle system. The first law of } \\
\text { thermodynamics: heat transfer and external work determine the change in } \\
\text { internal energy. }\end{array}$ & First questionnaire \\
\hline $\begin{array}{l}\text { Stage B- } \\
\text { Quantitative } \\
\text { treatment of } \\
\text { principles of } \\
\text { statistical } \\
\text { thermodynamics }\end{array}$ & $\begin{array}{l}\text { Meeting 7: Spatial distribution contributions to the entropy (which are termed } \\
\text { hereafter as spatial configuration entropy) are defined as the logarithm of } \\
\text { the multiplicity of the configurations of particles on a lattice. } \\
\text { Meetings } 8 \text { and 9: Energy distribution contributions to the entropy (which is } \\
\text { termed hereafter as energy distribution entropy) are defined as a logarithm } \\
\text { of the multiplicity of the configurations of energy quanta among particles. } \\
\text { The second law of thermodynamics is used to determine the equilibrium } \\
\text { energy states of objects in thermal contact. } \\
\text { Meeting 10: Free energy is defined as a reformulation of the entropy of the } \\
\text { Universe for systems in thermal contact with a heat reservoir. Free energy } \\
\text { minimization is shown to be equivalent to second law of thermodynamics. }\end{array}$ & $\begin{array}{l}\text { Second } \\
\text { questionnaire }\end{array}$ \\
\hline $\begin{array}{l}\text { Stage C-Analysis of } \\
\text { soft matter systems }\end{array}$ & $\begin{array}{l}\text { Meetings 11-13: Internal energy change and spatial configuration entropy } \\
\text { change are calculated for a model of a binary mixture of particles placed on } \\
\text { a lattice and combined in the free energy which is minimized to determine } \\
\text { phase separation for a given composition and temperature. } \\
\text { Meetings 14-19: Free energy is calculated and minimized in order to analyze } \\
\text { wetting of surfaces, capillary rise, and micellar self-assembly. }\end{array}$ & $\begin{array}{l}\text { Class discussion } \\
\text { transcript, third } \\
\text { questionnaire }\end{array}$ \\
\hline $\begin{array}{l}\text { Stage D_Inquiry } \\
\text { project }\end{array}$ & $\begin{array}{l}\text { Meetings 20-22: Free energy is calculated and minimized in order to predict } \\
\text { equilibrium configurations of polymers } \\
\text { Students performed independent experimental or simulational inquiry projects } \\
\text { that afforded them with an authentic scientific experience and allowed them } \\
\text { to further practice the ideas presented in stages A-C. }\end{array}$ & $\begin{array}{l}\text { Outside scope } \\
\text { of study }\end{array}$ \\
\hline
\end{tabular}

determine the change in the internal energy of the system, the change in the entropy of the system, and the change in the entropy of the surroundings during the process.

The sophistication of the scenario slightly increased in successive questionnaires: in the first questionnaire the context was that of a suspension of colloids that sediment; this was demonstrated in class. In the second, the context was that of a butanol/water solution-a less familiar set of fluids that, at room temperature, can mix for certain composition ranges and phase separate for others. The third context - that of milk-is more sophisticated since it included three components: fat globules, water, and casein protein, an emulsifier or "mixing agent" that stabilizes the fat globules in water and prevents the formation of a macroscopic "fat"/water equilibrium [43].

Since we later on analyze student answers vis-â-vis proper solutions, we describe in the following the conceptual resources needed to solve the phase separation problem. We define conceptual resources as elements of student knowledge that should be called on in order to solve the problem [44]. Although the original formulation of the resources framework identified resources as primitive ideas [45], here we follow Sayre and Wittman [46] and extend this to more "complex" resources that have an internal structure. In this perspective the main characteristic of a resource is not its primitivism but its durability, which manifests itself in the repeated use of the resource (or its fragility, which is demonstrated by only occasional use of the resource).

The resources needed to solve the phase separation problem are shown in Table III. The solutions of parts A and $\mathrm{B}$ of the question require invoking the definitions of internal energy (resource 1 in Table III) and entropy (resource 4). Each of these sets of knowledge elements is related to finer-grained scientific ideas (related to intermediate variables that are required to solve the problem) 


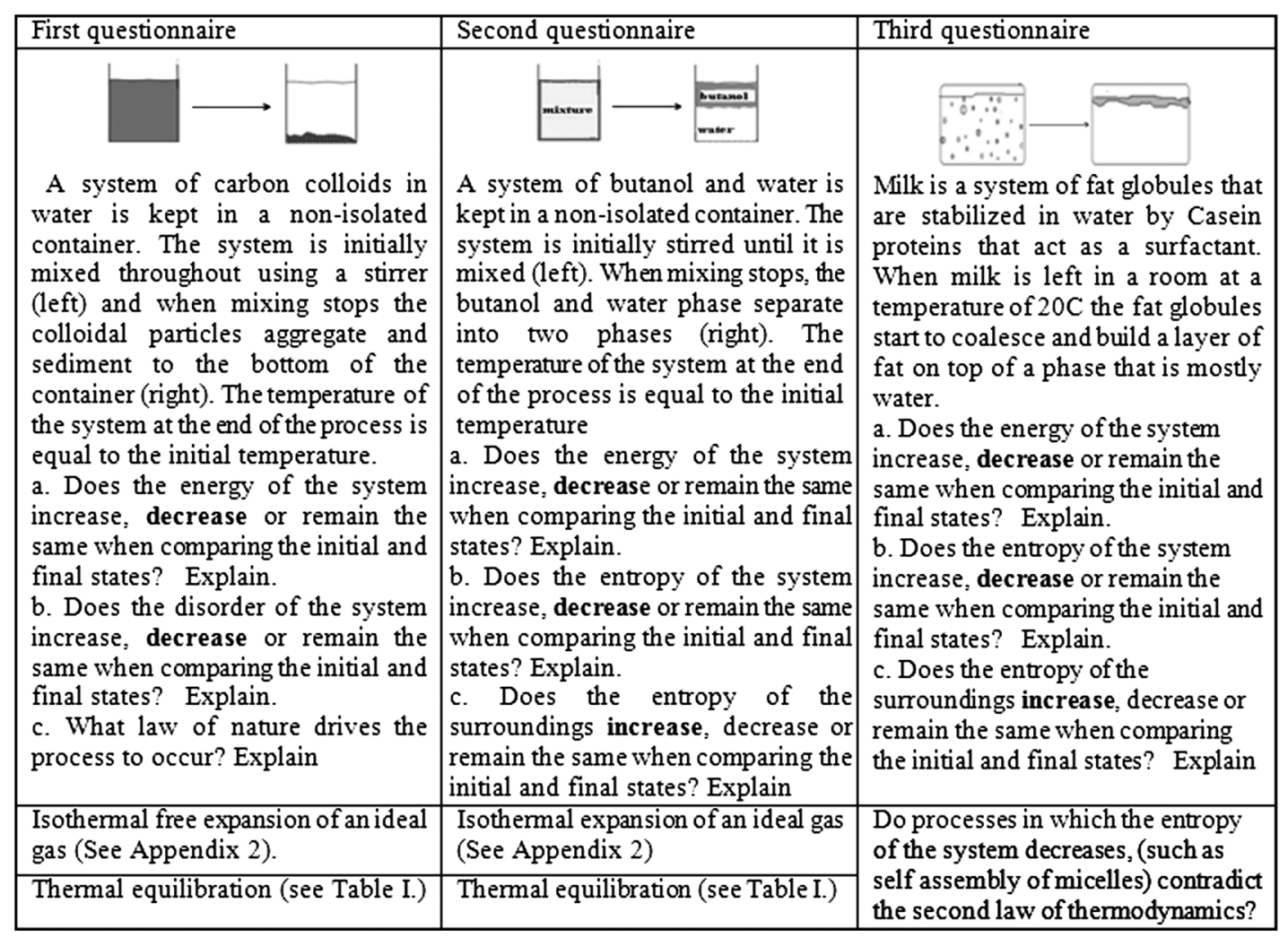

FIG. 1. The format of the three questionnaires. The correct predictions to the phase separation problem are marked in bold.

such as the average kinetic (resource 2) and potential energies (resource 3 ) that are the components of the internal energy. The student must first deduce from the problem statement the information that allows determination of the change in the values of these finer-grained scientific ideas that lead to predictions in the values of the target variables [48]. Table III summarizes the conceptual resources required to adequately solve the phase separation problem.

Part $\mathrm{C}$ of the problem requires integration of concepts. An adequate solution to the problem can be obtained via two paths: Path I turns part A of the problem into a resource (resource 7 in Table III) and integrates it with the principle of energy conservation that mandates an increase in the energy of the surroundings (resource 8). This path requires invoking an additional resource-that entropy is related to the number of configurations of energy quanta among particles (resource 4 in Table III), in order to conclude that the entropy of the surroundings increases.

Alternatively, one may take path II, in which part B of the problem turns into a resource (i.e., that the entropy of the system decreases) and is reconciled with the requirement of total entropy increase mandated by the second law of thermodynamics. The combined activation of these two resources leads to the conclusion that the entropy of the surroundings must increase in the process in order to compensate for the entropy decrease of the system. The two possible paths are illustrated in Fig. 2.

\section{Data analysis}

When tackling a conceptual problem such as the phase separation scenario presented above, students might associate their knowledge elements in a number of ways, some leading to scientifically acceptable statements and some not. We termed the association of the activation of specific resources and their application to the context of the problem solution paths. In order to capture both the appropriate and the alternative solution paths, we developed an analysis rubric that integrated both top-down and bottom-up approaches [49]. In the top-down approach we compared student responses to the questionnaire problems with the "proper solution path" discussed in the previous section. The comparison allowed us to determine which of the appropriate resources were invoked and whether these resources were properly applied. In addition, we also used a bottom-up approach to identify the various resources students employed as well as their management of both scientifically acceptable and other, unacceptable resources.

To achieve inter-rater reliability, three of the authors of this paper (S. A. S., E. Y., and E. L.) negotiated the emergent categories that described student thinking as reflected in their responses. The researchers reached complete agreement on the appropriate categories and the placement of student responses in the appropriate category.

The analysis of students' answers involves several modes of inference. The first mode consists of relatively 
TABLE III. Resources needed for proper solution of the phase separation problem.

\begin{tabular}{|c|c|c|c|}
\hline & & Resources & Application to problem \\
\hline $\begin{array}{l}\text { Part } \mathrm{A}- \\
\text { Determine } \\
\text { the change in } \\
\text { the internal } \\
\text { energy }\end{array}$ & & $\begin{array}{l}\text { (1) The internal energy of a system is a statistical } \\
\text { average of the sum of the particles' kinetic and } \\
\text { potential energies. } \\
\text { (2) The temperature is proportional to the average } \\
\text { kinetic energy of the particles in the system. } \\
\text { (3) The average potential energy of a system with } \\
\text { attractive, interparticle interactions decreases when } \\
\text { the particles in the system condense so that on } \\
\text { average they have stronger bonds with their } \\
\text { neighbors. }\end{array}$ & $\begin{array}{l}\text { I. Temperature is equal at the beginning } \\
\text { and the end of the process; therefore, } \\
\text { average kinetic energy does not change. } \\
\text { II. The interaction between the particles } \\
\text { is attractive and they condense; therefore, } \\
\text { potential energy decreases. The internal } \\
\text { energy, which thus decreases, combines } \\
\text { the fact there is no change in the average } \\
\text { kinetic energy (I) and that the average } \\
\text { potential energy decreases (II). }\end{array}$ \\
\hline $\begin{array}{l}\text { Part } \mathrm{B}- \\
\text { Determine } \\
\text { the change in } \\
\text { entropy of } \\
\text { the mixture } \\
\text { system }\end{array}$ & & $\begin{array}{l}\text { (4) Entropy is a measure of both the logarithm of } \\
\text { the multiplicity of the microscopic spatial } \\
\text { configurations of the system and of the distribution } \\
\text { of energy among these particles. } \\
\text { (5) When particles of a certain type occupy a smaller } \\
\text { volume in space, the number of possible spatial } \\
\text { configurations of the particles in the system } \\
\text { decreases. } \\
\text { (6) When the thermal energy in a system increases } \\
\text { and the number and type of particles is constant, } \\
\text { the entropy related to energy distribution increases. }\end{array}$ & $\begin{array}{l}\text { The spatial configuration entropy of the } \\
\text { system decreases since the particles of each } \\
\text { kind condense and occupy a smaller volume } \\
\text { in the mixture. The energy distributed among } \\
\text { the particles in the system does not change } \\
\text { since the temperature, that is a measure of the } \\
\text { thermal energy, remains constant. Since the } \\
\text { entropy of the system is the sum of spatial } \\
\text { configuration entropy and energy distribution } \\
\text { entropy, the total system entropy decreases. }\end{array}$ \\
\hline \multirow{2}{*}{$\begin{array}{l}\text { Part } \mathrm{C}- \\
\text { Determine } \\
\text { the change in } \\
\text { the entropy } \\
\text { of the } \\
\text { surroundings } \\
\text { [47] }\end{array}$} & Path I & $\begin{array}{l}\text { (7) The internal energy of the system decreases. } \\
\text { (deduced from part A). } \\
\text { (8) Any change in the internal energy of the system } \\
\text { implies a transfer of energy to or from the system } \\
\text { via heat or work (first law of thermodynamics). } \\
\text { (4) Entropy is a measure of the number of micro } \\
\text { scopic spatial configurations of the particles in the } \\
\text { system and the distribution of energy among these } \\
\text { particles. }\end{array}$ & $\begin{array}{l}\text { According to the first law of thermodynamics, } \\
\text { whenever the internal energy of the system } \\
\text { decreases, the energy of the surroundings } \\
\text { must increase. Since entropy is related to } \\
\text { the distribution of energy among the } \\
\text { particles (provided that the number of } \\
\text { spatial configurations of particles in the } \\
\text { surroundings does not change), the entropy } \\
\text { in the surroundings must increase. }\end{array}$ \\
\hline & Path II & $\begin{array}{l}\text { (9) The entropy of the system decreases (deduced } \\
\text { from part B). } \\
\text { (10) The second law of thermodynamics mandates } \\
\text { that, in spontaneous processes, entropy must } \\
\text { increase in the combined system plus surroundings. }\end{array}$ & $\begin{array}{l}\text { The entropy of the system decreases but the } \\
\text { total entropy of the universe must increase. } \\
\text { Therefore, the entropy of the surroundings } \\
\text { must increase more than the decrease in the } \\
\text { entropy of the system, so that the total } \\
\text { entropy increases. }\end{array}$ \\
\hline
\end{tabular}

straightforward inference of the resources students activated from their responses. Namely, delineating the solution paths and conceptual resources the students used in each stage. A second mode suggests explanations for the evolution in students' stability or fragility of the resource network over time [46]. Stable (but not necessarily scientifically accurate) resources have many connections to other resources, and their internal structure is unlikely to change under typical use. Fragile resources (termed "plastic resources" in Ref. [46]) are less durable in time or less stable in structure. We identify changes in the properties of resources according to their connections to prior knowledge, possible interference between conflicting pieces of knowledge [50], and resemblance to cognitive learning patterns in the acquisition of knowledge [51].
The third mode attempts to infer students' epistemological framing that governs their correlated management of several resources [14]. Student responses portray a uniquely defined aggregation of several activated knowledge resources which is activated by the individual's framing of the situation. Framing a situation means interpreting it and deciding how to act based on prior experience with similar situations. An epistemological framing is a construct of expectations that is unconsciously developed by students as they rationalizes experiences of acquiring knowledge from their past. For example, when students solve physics problems, we may infer that they frame physical knowledge as coherent if they reject a certain prediction that is not consistent with their other conceptual resources [52]. This epistemological framing is shaped by 
the students' interactions with their peers or teachers in previous encounters with physics problems. Framing physics as coherent is a useful epistemology; however, students' epistemological framings may also be detrimental to learning [14]. In this work, we interpret the overall evolution of the performance of case studies in terms of their epistemological framing, characterize the unproductive framings, and suggest some strategies for developing useful ones.

\section{FINDINGS}

\section{A. Students' approaches to analyzing changes in internal energy}

In stage $A$ of the curriculum, students learned to determine changes in internal energy by either analyzing changes in its components (the average kinetic and potential energies) or via the first law of thermodynamics. The first law of thermodynamics dictates that changes in internal energy can result from transfer of energy to or from the system via work or heat.

Section A of the problem asks the student to predict the change in the internal energy of the system that undergoes phase separation. The problem statement provides information regarding the particle configuration and temperature from which one can deduce that positive work is performed on the system by gravitational forces. However, no information is given regarding the direction of heat transfer; hence, students had to follow the first approach shown in Fig. 2 and analyze the change in internal energy as a sum of the changes in average kinetic and potential energy-related to the change in temperature and particle configuration, respectively.
The first case-study student, Ruben, combined both the "internal energy components" approach and the "firstlaw" approach. First, he analyzed the internal energy change by estimating the changes in kinetic energy of the molecules (constrained by the fact that temperature remained constant) to determine that the kinetic energy did not change "provided that the temperature of the system in both states remains exactly the same, we may thus conclude that the kinetic energy of the molecules in the system did not change." However, while he used kinetic energy, he did not activate the resource of change in the average potential energy due to the aggregation: "the kinetic energy of the molecules in the system did not change and therefore there is no change in the energy level of the system" [53]. We infer that in "energy level of the system" Ruben is referring to the internal energy; however, he does not relate the resource of internal energy to the potential energy, either because he does not associate it with the internal energy or because he does not realize that the change in configuration results in a modification of the potential energy. In the following section, we suggest how Ruben's background as a chemistry major might have led to this fragile manifestation of resources related to potential energy and its place in the internal energy.

Ruben did activate the "first-law" approach: "In addition, we did not invest work in the system." This assertion is correct except for work performed by the gravitational force on the system (unless he realized that gravitational work-which only determines the relative vertical positions of the two phases-is indeed negligible compared to the strength of the interparticle interactions). However, Ruben's first-law resource was incomplete, since in his

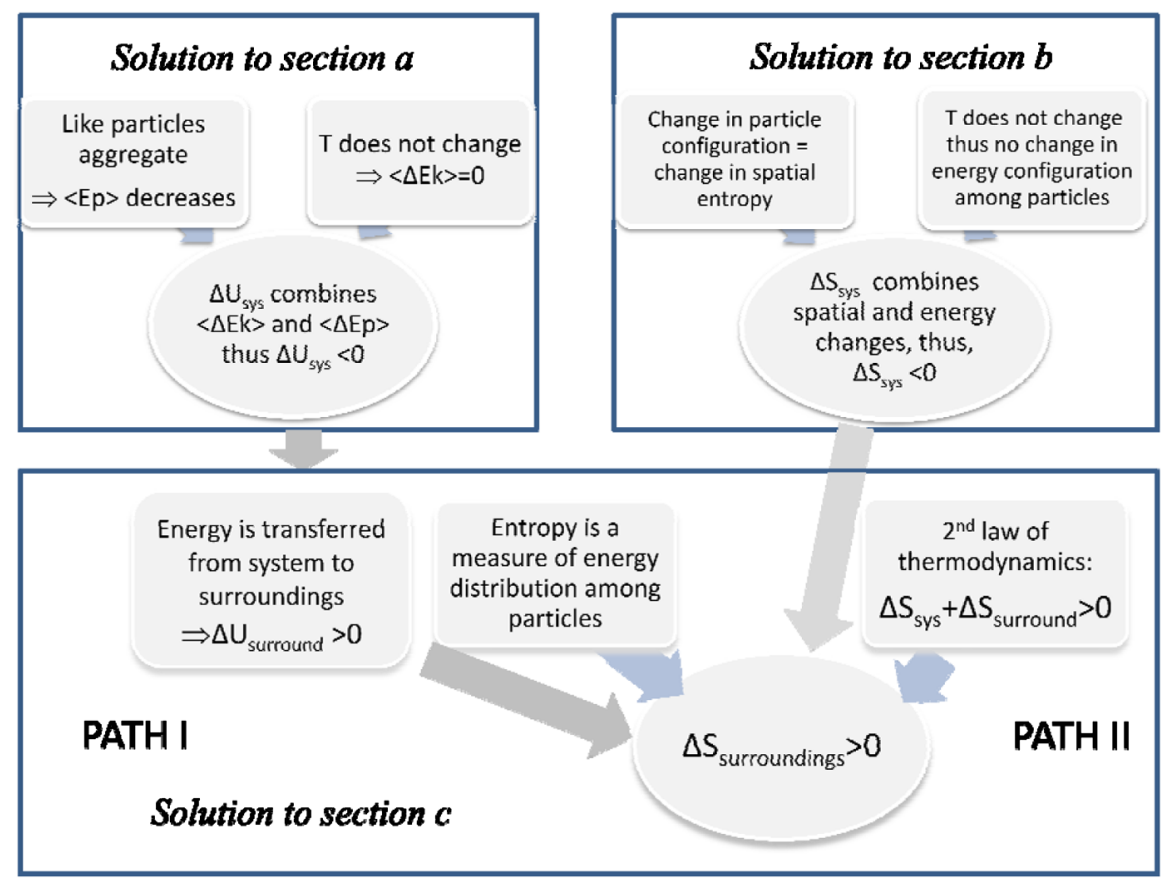

FIG. 2 (color online). Paths for proper solution of the phase separation problem. 
answer he did not invoke the effect of heat transfer on the change in the system plus reservoir energy [54]. Had he referred to this aspect he would have realized that the problem statement does not provide information regarding heat transfer, which excludes the use of the first-law approach. The term "in addition" in Ruben's answer suggests that he coordinates two alternative approaches to strengthen his suggested solution. Unfortunately, the apparent alignment of both approaches (which is scientifically incorrect) results from the fact that he activated only fragmented conceptual resources that led to the incorrect prediction by Ruben that the energy remains constant.

Simon took the only path that is relevant given the information in the problem statement-and used the resources relating changes in internal energy to possible changes in both the kinetic and potential energy (due to changes in particle configuration and temperature): "The total energy decreases: Regarding the thermal energy, it has not changed if we assume that already in the first state the temperature was equal to the surroundings. The carbon particles which are denser than the water sank, thus we may say that the center of mass is lower and therefore the gravitational energy is lower." However, Simon focused in his analysis on the (usually negligible) gravitational energy of the microsized particles as evidenced by their sedimentation and did not activate the change in potential energy related to their attractive interactions that drive their aggregation [55]. It might be that the problem scenario of sedimentation has triggered the resource of gravitational potential energy. However, it is also possible that because of Simon's physics background he was inclined to use gravitational potential energy, a topic that is thoroughly discussed in the standard high school physics curriculum [56] (but much less relevant to nano- or micron-scale soft matter).

Stage B incorporated a rigorous treatment of entropy, free energy, and the second law of thermodynamics in the context of ideal systems. In the second questionnaire that followed stage B, Ruben activated resources that better matched the information given in the problem and chose the scientifically acceptable "internal energy components" approach but abandoned the "first-law" approach he suggested in the first questionnaire. However, he still did not activate the resource related to the potential energy and hence concluded that the energy of the system remains constant during the process: "The energy of the system does not change from state I to state II since according to the data the kinetic energy of the molecules in state I and in state II does not change... and therefore the energy of the system does not change." Simon's explanation has also changed in the second questionnaire, where he acknowledged interparticle interactions as the main contribution to the change in internal energy [57]. However, it might be that the problem scenario in the second questionnaire, where no sedimentation takes place, was less likely to trigger activation of the resource of gravitation. "The attractive interactions increased and the repulsive interactions increased-thus the internal energy decreased" [58]. We infer that the discussions in stage B of the role of particle interactions in determining thermodynamic quantities, such as the internal energy, allowed both Ruben and Simon to relate the resource of energy to these more microscopic aspects.

Stage $C$ involved the analysis of phase separation and other soft matter phenomena calculated from minimization of free energy. However, this complex treatment was not required to answer the third questionnaire. Thus, it is possible that the material taught in this stage could interfere with students' ability to apply the basic concepts and principles of thermodynamics. In the third questionnaire that followed stage $\mathrm{C}$, Ruben acknowledged for the first time the contribution of interparticle interactions to the energy of the system: "Since the fat globules interacted with each other and created a layer with minimum free energy and the water molecules attract and lower their own free energy, as a result the energy of the system has decreased." However, he activates the resource of free energy (rather than the scientifically acceptable reference to potential energy) and does not mention the kinetic energy as he did before. He associates the decrease in free energy with the change in configuration related to the net interactions between fat globules and water molecules. In addition, it seems that he blends the knowledge resources of energy and free energy, confusing minimization of energy with the minimization of free energy [59]: "The energy of the system has decreased since in this process we see that the system has reached a more balanced state energetically ...." This tendency to interchange the resource of "minimal free energy" with that of energy can be explained in the accessibility of the free energy resource to Ruben given his prior knowledge as a chemistry student. Indeed, it is very similar to the literature finding regarding chemistry undergraduate students who stated that only exothermic processes in which the energy of the system decreases are spontaneous and that the system proceeds toward equilibrium because the enthalpy of the system is lower in equilibrium [18].

Simon, on the other hand, related the internal energy directly to the interactions: "The energy decreases: Particles that repel-water and oil, were separated and particles that attract got close to each other thus, the internal energy decreases." This analysis is much more concrete than was his response in the second questionnaire and expresses more clearly awareness to the complexity of the various interactions involved in the system.

\section{Summary and discussion}

The different approaches of Ruben and Simon at the various stages of the program are summarized in Fig. 3. The topology of the diagrams simulates those of the adequate solutions diagrams which were shown in Fig. 2. The 


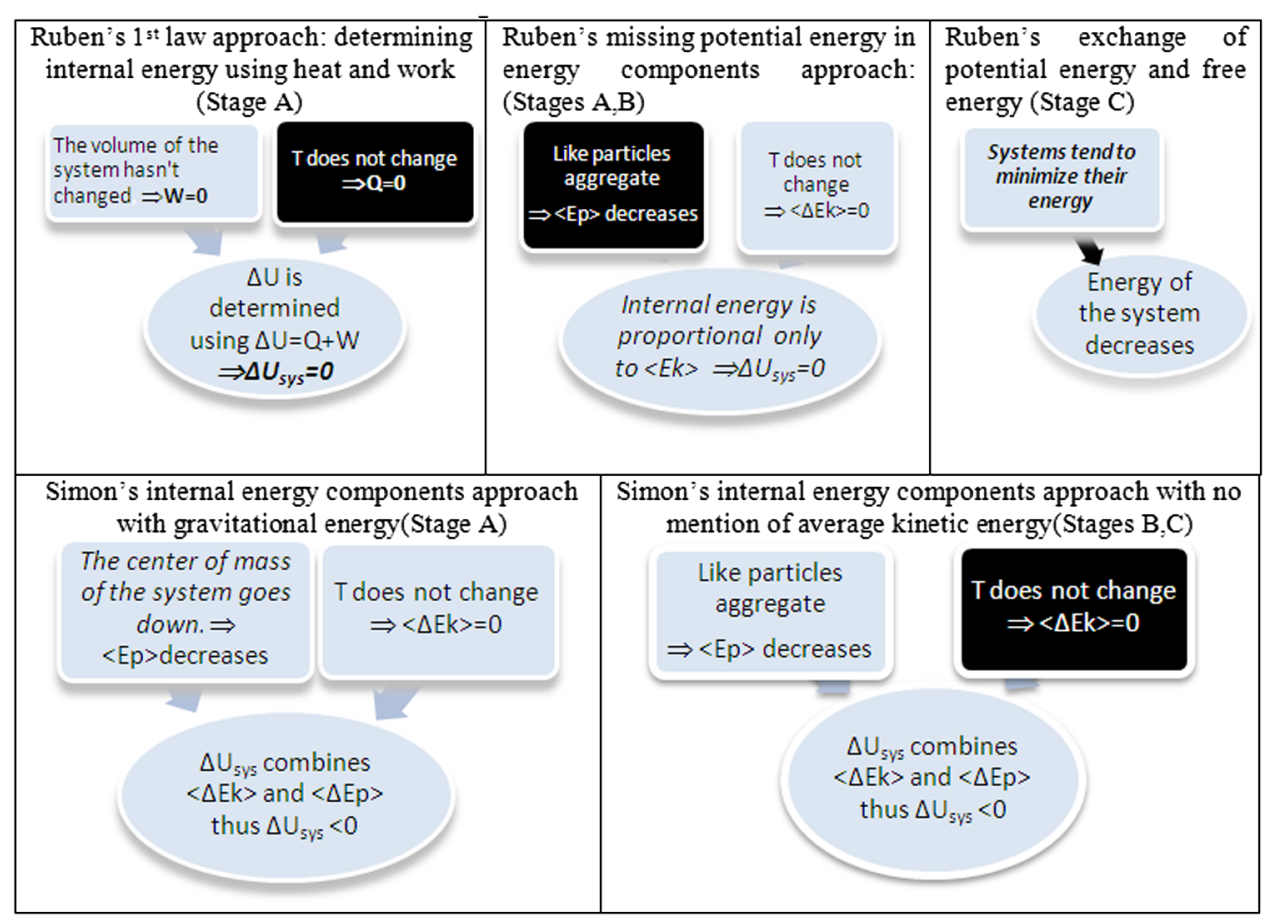

FIG. 3 (color online). Ruben's and Simon's various attempts at explaining the changes in internal energy. Missing resources are shaded in black, fragmented or partial resources are italicized.

findings show that throughout the program Simon managed the resources needed to analyze internal energy changes better than Ruben. In the first questionnaire, Simon carefully considered the information embedded in the problem scenario and chose the internal energy components approach, whereas Ruben tried several approaches and overlooked the lack of information that would disqualify the first-law approach. Even when Ruben used the proper internal energy components approach in the first and second questionnaires, he did not activate the resource of average potential energy, whereas Simon activated the connections between interactions and the change in potential energy. In the third questionnaire, given after the students were exposed to the use of the free energy to analyze several soft matter systems, Simon's responses did not incorporate this new resource, while Ruben blended the resource of free energy minimization into his response. However, free energy analysis was erroneously interpreted by him as associated with changes in the system energy related to interactions.

Ruben and Simon did not differ in their ability to cope with the mathematical level of the course (as manifested in their performance of quantitative tasks) or in their motivation to master the abstract concepts introduced in the program (as is evident from their involvement in initiating discussions intended to refine their understanding). In this paper, we take an interpretive perspective to explain students' activation of resources in terms of their prior experience as a chemistry or physics major. The Israeli physics curriculum devotes time to the analysis of potential energy and its relation to interactions in the 11th grade, in particular, in the context of gravitational forces. The chemistry curriculum in the 10th and 11th grades focuses on the periodic table (e.g., electronegativity, electron affinity), stoichiometry, chemical bonding, the gas laws, and oxidationreduction. The approach emphasizes diverse systems and phenomena. The topic of thermodynamics that includes a comprehensive discussion of energy changes in chemical processes (e.g., exothermic or endothermic reactions) appears only in the 12th grade curriculum. Accordingly, since this research took place prior to the presentation of energy in the chemistry curriculum, we hypothesize that Ruben's background as a chemistry major did not provide him with a stable potential energy resource that relates energy changes to interactions between particles. We also hypothesize that the unstable potential energy resource interfered with free energy resource which was repeatedly used in discussions of systems with interparticle interactions. This interference of the energy resources with free energy analysis might have also enforced Ruben's incorrect resource of energy minimization. In contrast, Simon's physics background led to the activation of the web of ideas related to potential energy, initially emphasizing gravitation, but later focusing on interparticle interactions. Examining this hypothesis could be the subject of a future study.

\section{B. Students' approaches to the analysis of entropy changes in the system and the surroundings}

Part B of the phase separation problem asked students to determine the change in entropy (disorder in the first questionnaire) of the system. The main resource required 
in part B is that of the spatial configuration entropy of the system. The entropy of the system is the logarithm of the multiplicity of particle configurations times the multiplicity of the kinetic energy distribution among the particles. The entropy related to the kinetic energy configurations does not change in the process (since the average kinetic energy is proportional to temperature which does not change), whereas the number of particle configurations decreases. Thus, when summing the contributions of the two components of entropy together, the entropy of the system decreases.

Already in the first questionnaire given after stage A of the curriculum, both Ruben and Simon correctly asserted that the disorder of the system decreases during phase separation in mixtures. However, their justifications reveal significant differences: Ruben related disorder to the number of spatial configurations and did not associate it with the energy distribution: "The disorder of the system decreases because the number of possible configurations of the carbon particles decreases when they aggregate and sink to the bottom of the vessel." On the other hand, Simon related disorder to collisions: "The disorder decreases because in the first state there are more collisions and interactions between the water and the carbon particles while in the second state they are separated." Relating disorder to the common types of collisions in the system (water-water or water-carbon particle) cannot be interpreted as associating disorder with energy distributions. However, this response indicates that Simon might be associating disorder with a dynamic, energy-related resource, which later may help him relate entropy to the energy distribution [60].

The second questionnaire was administered after stage B, which included a microscopic model of energy quanta used to calculate the entropy related to the energy distribution among the particles, in addition to the spatial configuration entropy. Ruben maintained and elaborated his perception of disorder as related to the number of spatial configurations in the system. This time he explained in detail the idea that the entropy is a measure of the possible configurations of the water and butanol molecules in the different states: "... In a state of different (separate) phases there is a decrease in entropy since there is a smaller and more limited place in which each molecule of the different materials can be found." In contrast to Ruben's description of the molecular picture, Simon did not activate the spatial entropy resource. Instead, he took a macroscopic approach and imposed the second law of thermodynamics on the system: "it increases because in a spontaneous process-entropy increases," erroneously asserting that the entropy in the system must increase as mandated by the spontaneity of the process, thereby creating a tautology. Simon's response reflects the tendency to apply the second law only to the system while dismissing the surroundings, as mentioned in the literature [18].
The third questionnaire was administered after stage C in which the instruction emphasized spatial configuration entropy (as opposed to energy distribution entropy) since the primary tool used was minimization of the Helmholtz free energy which is applicable only to isothermal processes [61]. In this questionnaire, Ruben's and Simon's answers were very similar: both considered only entropy related to spatial configurations. Ruben wrote, "The entropy of the system decreases ... We see that the fat globules arranged themselves in an organized state that caused the decrease in disorder and therefore a decrease in entropy," and Simon wrote, "The entropy decreases, there are less possible configurations."

To conclude, in the third and final questionnaire both case studies reached a similar understanding: that the change in the system entropy during phase separation is attributed to the decrease in spatial configuration entropy. However, Simon reached this conclusion after refining his approach from stage to stage and considering the second law of thermodynamics and energy distribution entropy (related to collisions or temperature), while Ruben retained his original understanding of entropy as solely related to spatial disorder; this did not equip him to answer the next section of the problem correctly.

Part $\mathrm{C}$ of the phase separation problem asked students to determine the change in the entropy of the surroundings. Part $\mathrm{C}$ of the problem was slightly different in the first questionnaire (instead of asking about the change in the entropy of the surroundings, the students were asked which law of nature dictates the process). Here, we will focus only on the responses to the second and third questionnaires. The changes in the properties of the surroundings can be estimated using two possible paths of reasoning (see Fig. 2) from changes in the system. Path I links the decrease in the internal energy of the system to an increase in the energy of the surroundings (via energy conservation). This increase in energy leads to an increase in entropy of the particles in the surroundings [62]. Path II uses the second law of thermodynamics to infer that for the total entropy of the Universe to increase, the entropy of the surroundings must increase to compensate for the decrease in the entropy of the system.

In his answer to part $\mathrm{C}$ in the second questionnaire, Ruben analyzed the changes in the entropy of the surroundings using the parameters of the surroundings itself: "The entropy of the surroundings does not change since there was no change in temperature or volume of the surrounding environment." This reasoning seems sensible, and only a very delicate understanding of the "heat bath" resource allows one to realize that it is incorrect. Namely, in the thermodynamic limit in which the system size relative to the reservoir size tends to zero, the entropy of the heat reservoir (the surroundings) can change even if its temperature remains the same (contrary to the system [63]). Ruben, possibly committed to an analysis in terms of the 
entropy of energy distributions, did not activate the second law of thermodynamics as a relevant tool that relates the entropy of the system to that of the surroundings [64]. When considering the interplay between the system and its surroundings, Ruben followed path I: "Indeed the entropy of the system has decreased, yet it does not affect the entropy of the surroundings since the system does not transfer energy or particles to the environment." He mentioned the possibility of energy transfer, but declined to pursue this option, probably because he incorrectly stated that the internal energy of the system remains the same. Thus, what stood in the way of a scientifically acceptable analysis of the problem by Ruben was his only partial activation of the "energy" resource, which we already identified in his answer to Sec. A of the problem. Finally, even though Ruben's analysis led him to an erroneous conclusion, we acknowledge once again his coordination of two pathways to solve the problem by coordinating two alternative approaches.

Simon, on the other hand, left his response to part $\mathrm{C}$ of the question (regarding the entropy of the surroundings) blank and told the teacher that he did not know the answer. $\mathrm{He}$ added that he felt uncomfortable making predictions since he was unsure of the physical principles. We interpret Simon's response in view of his answers, discussed earlier, to parts A and B. Although he could have used his answer to part A, where he realized the energy decrease in the system and thus could have followed path I (i.e., energy decrease in the system leads to change in the energy and entropy of the surroundings), he did not do so. We speculate that he perceived that the way to determine the change in entropy lay only in the use of the second law. Since he already used this tool in his answer to part B (where he associated the second law to the system per se, as he also did in his response to the thermal equilibration problem in the same questionnaire [65]), he was left with no other resources that he could activate to analyze changes in the surroundings.

In the third questionnaire, the answers to both part $\mathrm{C}$ of the phase separation problem as well as the additional problem required the activation of the second law resource. The additional problem was more direct as it asked students whether "processes in which the entropy of the system decreases (such as self-assembly of micelles) contradict the second law of thermodynamics." The relation between the second law of thermodynamics and free energy minimization was discussed in class in two separate lessons at this stage. One of these lessons included a task (see the Appendix) that required students to address the connection between the second law and the free energy analysis.

In his response to the third questionnaire, Ruben rejected the effect of the change in the system's energy on the surroundings even though he had considered it in the previous questionnaire: "The entropy of the surroundings does not change in the process since it isn't related to the process or influenced by it. The process occurs in the system spontaneously with no connection to the surroundings." We interpret this response as an activation of a deficient manifestation of path $\mathrm{I}$, in which the interplay between system and surroundings has been obscured. In addition, as before, Ruben still did not associate his prediction with the second law of thermodynamics; thus, path II was also not used. In contrast, Simon used both paths in his answer: he stated that the entropy of the surroundings increases because of the change in the system's energy (path I) and because of the second law of thermodynamics (path II): "The entropy of the surroundings increases for two reasons: a. the entropy of the system decreases and this decrease should be compensated. $b$. the energy of the system decreases, and the change in energy corresponds to the change in entropy."

The difference between the two case studies at this final stage is even more dramatic when viewed in light of their answers to the self-assembly problem. Here, Ruben explicitly revealed his view that the second law of thermodynamics may be violated and that it is applicable only to the system and does not include the surroundings: "Yes, (they contradict) since the second law of thermodynamics talks about the increase in disorder in the system .... But in systems in which the entropy decreases spontaneously the disorder decreases and it thus contradicts the second law." Ruben's response indicates that he did not perceive the second law of thermodynamics as one which is valid in this case: he thought that the law applies only to processes in which the entropy of the system increases, and does not apply to when the entropy of the system decreases [66].

Simon's response is quite opposite to that of Ruben: "No, the entropy of the system can decrease if the entropy of the surroundings has increased accordingly and sufficiently so that the total change in entropy (system + surroundings) will be positive." Simon's response demonstrates commitment to the second law as a binding principle and the ability to distinguish between the system and surrounding in order to resolve the apparent paradox between the entropy decrease of the system and the increase in the combined entropy of the combined system and reservoir as mandated by the second law.

The difference between Ruben and Simon was also evident in the following excerpt from a class discussion that took place in the third stage of the instruction. The discussion concerns an activity in which students were asked to match the components of the free energy (internal energy and entropy of the system) with those that enter the second law of thermodynamics (total entropy of the system and the surroundings) (see the Appendix for a full description of this activity).

(1) Teacher: Which item (in the free energy concept map) is related to the calculation of the entropy of the surroundings? 
(2) Simon: The internal energy

(3) Teacher: The internal energy? Why?

(4) Simon: Because it came from there

(5) Teacher: Why? How is this associated with the change of the entropy of the surroundings?

(6) Simon: Because energy, internal energy is, err ... if there is a change in energy then the energy has to go somewhere or to come from somewhere, and then we have a change in ... (stops)

While Simon activates the relation between the change in internal energy and the entropy of surroundings, he does not explain the mechanism in a clear manner. When the teacher prompts him to give a better explanation, he has difficulty formulating it and mentions that the process is related to a transfer of energy; however, he seems uncertain of his answer (line 6). The discussion then continues, and Ruben enters the scene:

(12) Teacher: (after the first discussion of the equivalence of the second law of thermodynamics and free energy minimization) So once again, how is the change in internal energy related to the entropy of the surroundings? Ruben, please explain.

(13) Ruben: Emm ... to tell the truth, the concept of internal energy is not so clear. I did not completely understand it. How is the change in internal energy related to the entropy of the surroundings?

(14) Teacher: Let's assume that the energy of the system changes, what happens to the energy?

(15) Ruben: If the energy has changed, it has of course influenced the surroundings. It can take energy from it or bring energy to it.

(16) Teacher: Yes, correct. And how is this related to entropy?

(17) Ruben: If the energy emmm ... of the internal energy has increased then, emm ... the entropy of the surroundings...

(18) Teacher: What happened to it? If the internal energy of the system has increased then the energy of the surroundings has decreased. Correct?

(19) Ruben: Yes.

(20) Teacher: And then, the entropy of the surroundings, what happens to it?

(21) Ruben: It also decreases. I think I have a problem with the concept of "internal energy"

(22) Teacher: Internal energy is the energy contained in the system. It is held within the system so to speak.

(23) Ruben: But I think we defined it as something else a few weeks ago... we used it in a different context.

Ruben seemed to follow the discussion led by the teacher, but complained he did not understand one of the main concepts involved in it - that of internal energy (line 21). This lack of understanding was also apparent in his analyses of the internal energy changes in Sec. A.
Ruben says that he is unsure of the relationship between the knowledge element of internal energy and the situation at hand. This might have later caused the association of free energy as a component of the internal energy that was evident from his response to part A. Unfortunately the discussion with the teacher, who did not realize how fragile Ruben's resource of internal energy is, did not serve to build this connection.

\section{Summary and discussion}

The findings of this section show that Simon gradually refined his management of knowledge resources concerning entropy and the second law of thermodynamics, whereas Ruben's reasoning deteriorated, especially during stage $\mathrm{C}$ of the curriculum. In their answers concerning the entropy of the system, both Ruben and Simon considered the contribution of spatial configuration entropy. However, Simon's answers reflected his awareness of a possible contribution of the entropy related to the kinetic energy distribution (related to collisions or temperature). We believe that this eventually allowed him to suggest that the resolution of the puzzle of the second law lay in the increased entropy of the surroundings to which heat had been transferred from the system (whose energy decreased).

A striking finding is the absence of the second law of thermodynamics from Ruben's responses. He predicted that the entropy of the surroundings does not change, even while the entropy of the system decreases. When the second law of thermodynamics was explicitly queried in the last problem with respect to processes in which the entropy of the system decreases, he explained that the second law contradicts these phenomena and is relevant only to processes in which the spatial configuration entropy of the system increases. Ruben's responses to the second questionnaire indicated that he was aware at that point of the possible interplay between the system and surroundings via an exchange of energy or particles. However, his response to the third questionnaire indicates that the interplay between them was no longer considered; instead he viewed the entire process as driven by forces within the system alone. In his response to part A of the third questionnaire concerning the change in the system's energy, he mentioned that the process in governed by a natural tendency of the system to reach a more balanced energy state.

In contrast, Simon made a stepwise progress towards expertlike reasoning regarding the phase separation phenomenon. Initially (in the second questionnaire), he invoked the second law of thermodynamics but did not acknowledge the interplay between the system and the surroundings. In the class discussion which followed the second questionnaire as well as in his responses to the third questionnaire, he was already able to reconcile the second law of thermodynamics with the entropy decrease in the system, by acknowledging the transfer of energy between 
system and surroundings that must increase the entropy of the surroundings.

What caused these dramatically different trajectories in the conceptual evolution of two students who were both committed and engaged? We first briefly examine a few explanations for this difference and the limitations of these explanations. We then demonstrate that interpreting the differences between Ruben and Simon in terms of their knowledge resources as well as in terms of the existence and stability or fragility of their epistemological resources yields the most appropriate explanation. As mentioned in the discussion of part $\mathrm{A}$ of the findings, the difference cannot be explained based on the students' motivation or level of mathematics, nor can it be explained based on the teaching in the program, since both students were exposed to same lessons and activities.

The first attempt to explain the difference between the case studies is related to the stability of conceptual resources regarding energy. We pointed out that Simon's potential energy resource was either inappropriately applied or initially unstable as reflected by his identification of gravitational potential energy (as opposed to the interparticle interactions) in the analysis of phase separation in the first questionnaire. After this manifestation of a nascent resource of potential energy, Simon did use the energy resources in a consistent and stable manner in the second and third questionnaires. Simon's integration of the energy ideas into a stable network of resources enabled the resolution of the contradiction between the entropy decrease in the system and the overall increase in entropy as expressed in his answer to the third questionnaire. In contrast, Ruben did not activate the potential energy resource in any of the questionnaires. Moreover, he acknowledged being unsure of his understanding of the term "internal energy" in the classroom discussion. His fragile network of ideas suffered from the introduction of the free energy resource (which has as its prerequisite a good understanding of internal energy and its relation to potential energy) which interfered with the other energy-related resources. This created a stable but incorrect resource of energy minimization and disabled the activation of a resources related to energy exchange between the system and the environment in the third questionnaire. However, this interpretation does not explain why Ruben thought that the second law of thermodynamics could be violated in the context of phase separation and self-assembly.

In order to provide a more satisfying explanation of the difference in the activation of the resource related to the second law of thermodynamics, we interpret the difference between the two students in terms of their epistemological framings. In particular, we refer to two epistemological aspects: the first is related to integration of the knowledge elements and the expectation for coherence. Redish and Hammer [52] explain seeking coherence as the tendency to resolve conflicts between various ideas and findings, rather than thinking of one's physics knowledge as a set of unrelated facts and formulas. However, the epistemological resource of seeking coherence is insufficient for creating a stable net of ideas. For example, we suggest that Ruben was looking for coherence in his attempts to verify his answers by cross-checking them with other approaches or solutions paths. This was evident in his response to part A concerning energy in the first questionnaire and part $\mathrm{C}$ in the second. It also emerged in the excerpt from the class discussion where he says that he is not confident in his understanding since he does not know how to associate internal energy with the entropy of the surroundings. However, Ruben's search for coherence yields a constantly changing management of resources which resulted in erroneous scientific explanations.

We suggest that the dramatic difference between Ruben and Simon lies in another manifestation of coherence: adherence to fundamental principles or foothold ideas that should be valid under any circumstances and with no exceptions. In this epistemological resource, one chooses a principle as an anchor for associating a coherent set of resources. Accordingly, one refines these fundamental ideas so that they fit different situations, and question contradictory results, rather than relinquishing the validity of the foothold ideas themselves. By asking about the change in entropy of the surroundings, the phase separation scenario (in the second and third questionnaires) indirectly examines the extent to which students perceive the second law of thermodynamics as a foothold idea. Students, who frame the second law as a foothold idea should have used it to monitor whether their prediction regarding the change in the entropy of the system plus surroundings is indeed consistent with it. The perception of the consistency of a phenomenon with a foothold idea was gauged directly in the self-assembly problem (third questionnaire), that required students to reason about an apparent contradiction of the second law and the decrease in the system entropy. Indeed, Ruben's responses clearly demonstrated that he did not consider the second law of thermodynamics (that he perceived to apply only to the system) as a foothold idea that is always valid. This framing placed the focus of Ruben's reasoning on the phenomenology, and to his "flexible approach" in which physical laws can be adjusted depending on the situation.

In contrast, Simon adhered to the physical principle of the second law of thermodynamics. We suggest that Simon's activation of the conceptual resource that links the system and surroundings in his responses to the third questionnaire followed a need to refine the principle of adherence to the second law of thermodynamics after his failure to reconcile it with the problem situation in the second questionnaire. Indeed, Simon's search for refinement enabled him to refocus his analysis from the system alone to invoke the interplay between the system and its surroundings in the third questionnaire. Ruben's 
deterioration can be explained in a similar manner. Ruben did not activate the conceptual resources needed to clarify the interplay between system and surroundings because he did not frame the contradiction between his understanding of entropy as spatial disorder (of the system) and the second law of thermodynamics as a problematic point which needed refinement.

The majority of students who stated that processes in which the entropy of the system decreases contradict the second law of thermodynamics were chemistry majors. This may hint that their background might have shaped their epistemological framing. Israeli chemistry majors are introduced to a multitude of systems and phenomena during their 10th and 11th grade studies. These ideas are not "derived" from a set of unified explanatory rules as noted by several educators. For example, Levy-Nahum et al. [67] point out that in many textbooks ionic interactions are termed ionic bonds, while van der Waals interactions are termed van der Waals forces although both originate from electrostatic attractions. This example indicates that a student might lack a unified perspective of ideas of bonding and interactions, which may lead to a less generic and coherent epistemological framing in which the student perceives that basic physics principles are binding only in specific circumstances. However, this claim can also be correct with respect to the physics curriculum in which force laws may seem different when the context changes, for example, from mechanics to electricity and magnetism [1]. Still, the physics curriculum emphasizes a small set of physical laws, while the chemistry curriculum does not. This may explain the difference in the cultivation of the epistemological resource of foothold ideas between physics and chemistry majors.

\section{IMPLICATIONS}

Our study has implications for the design of curricula in which introductory students are required to analyze, via statistical thermodynamics, systems with interparticle interactions. We now outline and emphasize three such implications. First, our findings indicate the need for a more thorough treatment of the energy-related concepts in stage A of the course. Originally, the course included two meetings in which the students were reminded of energy-related concepts that were previously introduced to them as part of their middle school curricula and which is further elaborated in the course of the physics and chemistry high school curricula. Nevertheless, Ruben's responses (and those of other students as well) show that this limited treatment equipped only a minority of the students (probably only those with a physics background) with linked conceptual resources that allowed the linkage, in a scientifically acceptable manner, of the spatial configuration, interactions, potential energy, and internal energy. In order to establish potential energy as a useful resource, its introduction using the first law of thermodynamics according to Arons [15] should be further supported, possibly by tutorials such as those proposed by Lindsey et al. [20] Also, we believe that Simon's response to the first questionnaire problem on the sedimentation of coal particles indicates that it is important to expound on the knowledge resource related to gravitation and to explicate the relative effects of gravity and of interparticle interactions in the phase separation process.

Second, in order to give additional support to students such as Ruben, whose epistemological framing is inappropriate in its application to fundamental physical laws that should serve as foothold ideas, we endorse the suggestion of Redish and Hammer to make the epistemology explicit in the curriculum [52]. The concept maps tools used in the program explicated that the second law of thermodynamics is a principal law of nature that must be satisfied in each and every equilibrium process. However, this aspect was not elicited and discussed in students' tasks and following classroom discussion. Thus, one should provide opportunities to discuss the preference of various resources over others; that is, what should one do if a scenario activates two conflicting resources? How are the resources aligned with scientifically acceptable interpretations of foothold ideas? How can they resolve apparent mismatches of foothold ideas in the analysis of actual phenomena?

This may be achieved, for example, by discussing with the students the resource of energy minimization that they have activated. The teacher can ask students whether they find this idea to be a foothold idea and whether it relates to other foothold ideas they might be aware of, such as energy conservation and free energy minimization that parallels the second law of thermodynamics for systems that are in contact with a heat reservoir.

Third, this study should raise the reader's awareness of the value and risks of using a spiral curriculum. Namely, while discussions of the same concepts in different contexts with a gradually increasing level of sophistication provide opportunities for students to improve their understanding of earlier concepts, this approach can also deteriorate the understanding of students like Ruben whose network of resources is fragile.

Finally, we note that the statistical thermodynamics approach used by us and others indeed treats the particle configurations and interactions within the system in a manner that provides "microscopic" physical insight into the system thermodynamics; however, the surroundings are commonly treated thermodynamically in terms of heat transfer and the resulting entropy increase. No microscopic model of the surroundings is usually discussed; had this been done, it would perhaps help students develop a more full mental representation of the statistical-thermodynamic treatment of the configurations, interactions, and heat transfer of both the systems and its surroundings and allow students to resolve the apparent contradiction of the 
entropy decrease in phase separation with the second law. The design of such a complete statistical model and its incorporation in the teaching of phase separation is an interesting and possibly useful future project.

We believe that incorporating these instructional tools may enable more students like Ruben who possess genuine, intrinsic motivation to know more about science to cope with the challenge of current interdisciplinary topics in high school or in introductory level college courses.

In addition, this case-study research provides initial information on the learning of fundamental principles in a sophisticated context. Specifically, this study provides initial hypotheses regarding student learning that may be tested and refined in large-scale research. For example, our hypothesis regarding the role of a chemistry background when studying systems from a physics perspective may contribute to studies of the crossing of epistemological boundaries as suggested by Watkins et al. [2]

\section{ACKNOWLEDGMENTS}

The course on soft matter is a collaboration of the School of Contemporary Science at the Davidson Institute of Science Education and the Department of Science Teaching at the Weizmann Institute of Science. S. A. S. is grateful to the Israel Science Foundation and the Schmidt Minerva Center as well as to the Perlman family Foundation for their historic generosity and support of science. We wish to thank Avi Golan, Avi Hofstein, and Bat-Sheva Eylon for their ongoing support of the program, Yehuda Roth, Ron Blonder, Benny Geiger, and Anat Yarden for assistance in the initial stages of the project, and David Andelman, Ernesto Joselevic, and Jacob Klein for guest lectures. Most importantly, we thank the high school students who participated in the soft matter course, especially Ruben and Simon for the pleasure of teaching them and learning from them so much.

\section{APPENDIX}

\section{Soft matter curriculum: Brief description}

Stage A starts with demonstrations of unusual materials (for example, silly putty and emulsions) whose state of matter is not simply liquid, gas, or solid (meetings 1 and 2). The demonstrations are used to motivate the use of an atomistic approach [7] as a necessary link between the microscopic picture and the macroscopic properties of many-particle systems. A microstate of a system can be envisaged as snapshots that captures the instantaneous particle positions and velocities. A macrostate is characterized by the value of a macroscopic property (for example, the average particle orientation in a liquid crystal and the volume occupied by a polymer chain with $N$ segments) averaged over many microstates.

Meetings 3 and 4 are devoted to the statistical ideas needed to link the microscopic picture with the macroscopic properties of a system, including probability distributions, their mean, and standard deviation. All of those concepts are demonstrated by the toss of a coin or a die [68]. The concept of "disorder" is then defined as a precursor for a later discussion of entropy. Disorder is defined as a measure of the number of microstates that correspond to a certain macrostate. The second law of thermodynamics is introduced in terms of the tendency of an isolated system to spontaneously increase its disorder. This approach is taken despite recommendations against the use of this term [23] because spatial configuration entropy is central to the analysis of soft matter systems.

The presentation continues with an examination of disorder in systems in contact with a heat reservoir (for example, ice in a freezer), where the disorder of system decreases, because energy is dissipated to the surroundings. Such phenomena pave the way for the introduction of the energy of multiparticle systems in meetings 5 and 6. Several educators recommended presenting energy in terms of a system of several bodies using the first law of thermodynamics [15]. In such contexts, internal energy is defined as a combination of the average kinetic energy and the average potential energy (interactions) of the particles in the system. The change in potential energy is deduced from the negative work performed on the particle by internal, conservative forces [8] and is applied to the analysis of the interaction between two magnets. The first law of thermodynamics is shown to determine how systems exchange energy with their surroundings and the energy transferred from a system to the surroundings or vice versa is interpreted as a change of the disorder of energy in the surroundings. The stage is concluded with an experiment on colloidal aggregation [69] which is used to motivate a quantitative model for predicting the macrostate of a system.

Stage B spans four meetings and entails a quantitative presentation of entropy and the second law of thermodynamics. Entropy was introduced first in meeting 7 as a quantitative measure of "spatial disorder" using a lattice as a representation of both ideal gases (particles and

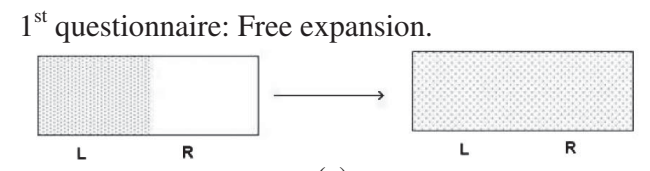

(a)

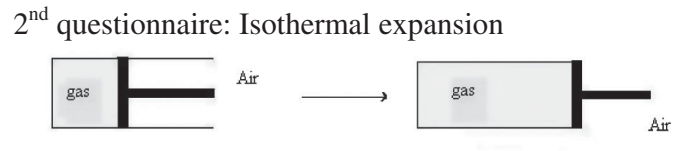

(b)

FIG. 4. (a) Free expansion of an ideal gas. (b) Isothermal expansion of an ideal gas. 
TABLE IV. A comparison of student correct answers to the ideal gas problem.

\begin{tabular}{|c|c|c|c|c|}
\hline & $\begin{array}{l}\text { Soft matter students } \\
\qquad(N=14) \text { first } \\
\text { questionnaire }\end{array}$ & $\begin{array}{l}\text { College- } \\
\text { Preinstruction } \\
\quad(N=20)\end{array}$ & $\begin{array}{l}\text { Soft matter students } \\
(N=11) \text { second } \\
\text { questionnaire }\end{array}$ & $\begin{array}{l}\text { College- } \\
\text { Postinstruction } \\
\quad(N=16)\end{array}$ \\
\hline (b) Entropy of the system increases & $100 \%$ & $75 \%$ & $100 \%$ & $94 \%$ \\
\hline $\begin{array}{l}\text { (c) Entropy of the surroundings remains } \\
\text { unchanged in the free expansion scenario } \\
\text { illustrated in Fig. 4(a) and decreases in the } \\
\text { isothermal expansion scenario of Fig. 4(b) }\end{array}$ & not applicable [47] & $60 \%$ & $73 \%$ & $94 \%$ \\
\hline
\end{tabular}

vacuum) and ideal mixtures (two types of particles, with no interactions). The advantage of the lattice model is that its visual representation supports the rather abstract mathematical derivation [70]. Next, in meetings 8 and 9 entropy is introduced as the logarithm of the number of possible microstates of energy quanta in a system of $N$ particles [71]. The second law of thermodynamics is then used to determine the equilibrium energy distribution of two bodies in thermal contact and the statistical definition of temperature $\frac{1}{T}=\frac{d S}{d Q}$ is derived (adapted from Ref. [25]). Spatial configuration entropy and energy distribution entropy are then summed to quantify the total entropy of the system. Free energy is introduced in meeting 10 as a mathematical tool which is equivalent to the total entropy of the system and its surroundings. Minimization of the free energy, equivalent to the maximization of the total entropy [72], can be used to determine if a process is spontaneous based only on the changes of the properties of the system (internal energy and entropy); calculating the changes in the entropy of the surroundings is thus not necessary.

Stage C spans eight meetings and encompasses the analysis of various soft matter systems using free energy minimization. This stage starts with the analysis of phase separation in binary mixtures represented using a lattice (meetings 11-13). The lattice construction facilitates the calculation of the spatial entropy of a mixture with a given composition as well as an approximation for its internal energy [73]. The free energy includes the changes in internal energy and entropy; its minimization yields a phase diagram which is used to determine the equilibrium state of the system. The last five meetings of this stage (meetings 14-18) present the free energy analysis of surface-related phenomena and surfactant self-assembly. The full derivation of the phase separation analysis as well as the analyses of other soft matter phenomena was discussed in detail in a separate paper [5].

The curriculum was developed via a design-based approach in which preliminary analysis of the instruction was used for revisions of the teaching materials. These revisions allowed us to refine the presentation of the underlying concepts and principles into a coherent, continuous curriculum for which a more comprehensive description can be found in a separate paper [5].

\section{Ideal gas question adapted from Ref. [42]}

A system consisting of an ideal gas goes through an expansion processes as shown above. The initial and final temperatures $(T)$ are the same for each process. (See Fig. 4 and Table IV.)

(a) Does the internal energy of the system increase, decrease or remain unchanged?

(b) Does the entropy of the system increase, decrease or remain unchanged

(c) Does the entropy of the surroundings increase, decrease or remain unchanged?

\section{Worksheet activity related to the second law of thermodynamics}

The worksheet problem used a concept map that summarized the presentation slides and linked between free energy and the entropy of the surroundings. The worksheet was followed by a class discussion conducted at the beginning of the stage (see Fig. 5).

(a) Fill in the concept map which describes the theoretical analysis of the phase separation process

(b) Which rectangle is related to the change in the entropy of the surroundings?

(c) Which rectangle reflects the activation of the 2 nd law of thermodynamics (Use presentation of lecture 12 slide 2).

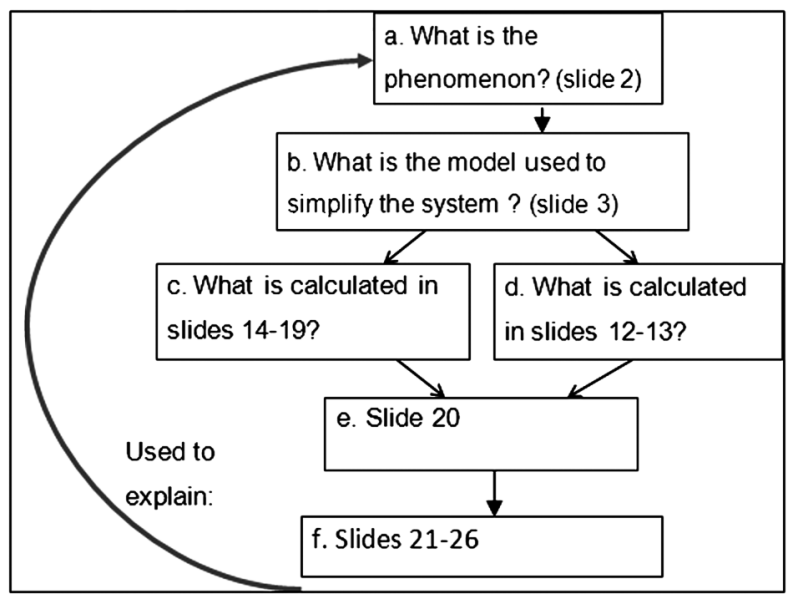

FIG. 5. Concept map presented in the worksheet. 
[1] E. Bagno and B. Eylon, From problem solving to a knowledge structure: An example from the domain of electromagnetism, Am. J. Phys. 65, 726 (1997).

[2] The difference between physics courses and biology courses is discussed in J. Watkins, J.E. Coffey, E.F. Redish, and T.J. Cooke Disciplinary authenticity: Enriching the reforms of introductory physics courses for life-science students, Phys. Rev. ST Phys. Educ. Res. 8, 010112 (2012).

[3] D. Halliday, R. Resnick, and J. Walker, Fundamentals of Physics (John Wiley and Sons, New York, 2005), 7th ed., p. 509.

[4] R. A. L. Jones, Soft Condensed Matter (Oxford University Press, Oxford, England, 2002); M. Kleman and O. Lavrentovich, Soft Matter Physics-An Introduction (Springer, New York, 2003); S. A. Safran, Statistical Thermodynamics of Surfaces, Interfaces and Membranes (Westview Press, Boulder, 2003).

[5] E. Langbeheim, S. Livne, S. Safran, and E. Yerushalmi, Introductory physics going soft, Am. J. Phys. 80, 51 (2012).

[6] The level of the content in these subjects is equivalent to advanced placement courses in the U.S. We note that less than $10 \%$ of Israeli high school students choose to major in more than one science subject. As a result most chemistry students do not take an introductory physics course and most physics students do not take introductory chemistry courses.

[7] F. Reif Thermal physics in the introductory physics course: Why and how to teach it from a unified atomic perspective, Am. J. Phys. 67, 1051 (1999).

[8] R.W. Chabay and B. A. Sherwood, Matter and Interactions: Modern Mechanics (John Wiley and Sons, New York, 2011), 3rd ed., Vol I, pp. 472-488.

[9] J. Petri, H. Niedderer, A learning pathway in high-school level atomic physics, Int. J. Sci. Educ. 20, 1075 (1998).

[10] E. Etkina, T. Matilsky, and M. Lawrence, What can we learn from pushing to the edge? Rutgers Astrophysics Institute motivates talented high school students, J. Res. Sci. Teach. 40, 958 (2003).

[11] The mean-field entropy of the mixed system is generally higher than the combined entropies of the more condensed and the more dilute systems that coexist in equilibrium phase separation.

[12] M. Sözbilir and J. M. Bennett, A study of Turkish chemistry undergraduates' understandings of entropy, J. Chem. Educ. 84, 1204 (2007).

[13] J. Barak, M. Gorodetsky, D. Chipman, and B. Gurion, Understanding of energy in biology and vitalistic conceptions, Int. J. Sci. Educ. 19, 21 (1997).

[14] D. Hammer, A. Elby, R. Scherr, and E. F. Redish, in Transfer of Learning from a Modern Multidisciplinary Perspective, edited by J. Mestre (Information Age Publishing, Greenwich, CT, 2005) pp. 89-119.

[15] A. B. Arons, Development of energy concepts in introductory physics courses, Am. J. Phys. 67, 1063 (1999); R. W. Chabay and B. A. Sherwood, Modern mechanics, Am. J. Phys. 72, 439 (2004); J. W. Jewett, Energy and the confused student I: Work, Phys. Teach. 46, 38 (2008); J. W. Jewett, Energy and the confused student II: Systems, ibid. 46, 81 (2008).
[16] G. Erickson, in Children's Ideas in Science, edited by R.E. Driver, E. Guesne, and A. Tiberghien (Open University Press, Philadelphia, PA, 1985), p. 55.

[17] D. E. Meltzer, Investigation of students' reasoning regarding heat, work, and the first law of thermodynamics in an introductory calculus-based general physics course, Am. J. Phys. 72, 1432 (2004).

[18] P.L. Thomas and R.W. Schwenz, College physical chemistry students' conceptions of equilibrium and fundamental thermodynamics, J. Res. Sci. Teach. 35, 1151 (1998).

[19] M.E. Loverude, C. H. Kautz, and P. R. L. Heron, Student understanding of the first law of thermodynamics: Relating work to the adiabatic compression of an ideal gas, Am. J. Phys. 70, 137 (2002).

[20] B. A. Lindsey, P. R. L. Heron, and P. S. Shaffer, Student ability to apply the concepts of work and energy to extended systems, Am. J. Phys. 77, 999 (2009).

[21] B. A. Lindsey, P. R. L. Heron, and P. S. Shaffer Student understanding of energy: Difficulties related to systems, Am. J. Phys. 80, 154 (2012).

[22] T. J. Greenbowe and D. E. Meltzer, Student learning of thermochemical concepts in the context of solution calorimetry, Int. J. Sci. Educ. 25, 779 (2003).

[23] See, for example, F. L. Lambert, Disorder-A cracked crutch for supporting entropy discussions, J. Chem. Educ. 79, 187 (2002); H.S. Leff, Thermodynamic entropy: The spreading and sharing of energy, Am. J. Phys. 64, 1261 (1996).

[24] See, for example, A. Ben-Naim, Entropy: Order or information, J. Chem. Educ. 88, 594 (2011); R. H. Swendsen, How physicists disagree on the meaning of entropy, Am. J. Phys. 79, 342 (2011).

[25] This treatment can be found in Refs. [7,8] as well as in R. Baierlein, Entropy and the second law: A pedagogical alternative, Am. J. Phys. 62, 15 (1994); T. Moore and D. V. Schroeder A different approach to introducing statistical mechanics, Am. J. Phys. 65, 26 (1997); D. C. Schoepf, A statistical development of entropy for the introductory physics course, Am. J. Phys. 70, 128 (2002).

[26] P. W. Atkins, The 2nd Law (Scientific American Books, New York. 1984); R. Chang, General Chemistry (McGraw-Hill College, New York, 1998), 6th ed.

[27] L. Boltzmann, Lectures on Gas Theory, translated by S. G. Brush (University of California Press, Berkeley, CA, 1964), pp. 442-443.

[28] F. L. Lambert, Shuffled cards, messy desks, and disorderly dorm rooms-Examples of entropy increase? Nonsense!, J. Chem. Educ. 76, 1385 (1999).

[29] Entropy was presented as the number of ways in which energy can be distributed in a system.

[30] E. M. Carson and J. R. Watson, Undergraduate students' understandings of entropy and Gibbs free energy, Univ. Chem. Educ. 6, 4 (2002) [http://www.rsc.org/pdf/ uchemed/papers/2002/p2_carson.pdf].

[31] W. M. Christensen, D.E. Meltzer, and C. A. Ogilvie, Student ideas regarding entropy and the second law of thermodynamics in an introductory physics course, Am. J. Phys. 77, 907 (2009).

[32] It is important to note that introductory physics courses mostly use standard general physics textbooks. For 
example, the students that participated in the heat engine study Ref. [33] used the textbook Fundamentals of Physics (Ref. [3]) which presents the concepts of entropy in a nonstatistical, traditional fashion using the Clausius definition.

[33] M. J. Cochran and P. R. L. Heron, Development and assessment of research-based tutorials on heat engines and the second law of thermodynamics, Am. J. Phys. 74, 734 (2006).

[34] For example, students may identify a process in which heat is fully converted into useful work as a possible process because it does not contradict the first law of thermodynamics. However, this type of process is impossible according to the second law of thermodynamics since the total entropy does not increase.

[35] A. C. Banerjee, Teaching chemical equilibrium and thermodynamics to undergraduate general chemistry classes, J. Chem. Educ. 72, 879 (1995).

[36] P. L. Thomas, Student conceptions of equilibrium and fundamental thermodynamic concepts in college physical chemistry, Ph.D. thesis, University of Northern Colorado, 1997 [Publication No. AAI9729078, ISBN: 9780591382501].

[37] The study related to a unique program can be found in Ref. [9]. Two studies concerning traditional curricula are L. Lising and A. Elby, The impact of epistemology on learning: A case study from introductory physics, Am. J. Phys. 73, 372 (2005); K. S. Taber, Exploring conceptual integration in student thinking: Evidence from a case study, Int. J. Sci. Educ. 30, 1915 (2008).

[38] Note that each meeting in our high school course is 2.5 hours long. The format of the meetings in the program is quite different from the college lecture or recitation format. The lectures are intertwined with the recitations and labs and involve discussions in which students raise their own questions and ideas. Moreover, since the course took place in an afternoon, out-of-school setting, homework requirements were minimal and practice exercises took place during the meetings. As an outcome, the pace was much slower than that of a college lecture course. As a rough estimate, we suggest that each 2.5 hour meeting might be equivalent to $3 / 4$ hour lecture and $1 / 2 \mathrm{hr}$ of recitation in a college setting.

[39] National Committee for Science Education Standards and Assessment, NRC, National Science Education Standards (National Academies Press, Washington, DC, 1996). The content standards for grades 5-8 include, "Heat moves in predictable ways, flowing from warmer objects to cooler ones, until both reach the same temperature" (p. 155). The content standards for grades 9-12 include, "The total energy of the universe is constant. Energy can be transferred by collisions in chemical and nuclear reactions, by light waves and other radiations, and in many other ways. However, it can never be destroyed. As these transfers occur, the matter involved becomes steadily less ordered" (p. 180).

[40] For some of the proportions that were too small for the normality condition, we used the Wilson interval test; see L.D. Brown, T. T. Cai, and A. DasGupta Interval estimation for a binomial proportion, Stat. Sci. 16, 101 (2001) [http://projecteuclid.org/DPubS?service=UI\&version $=1.0 \&$ verb=Display\&handle=euclid.ss/1009213286].
[41] These criteria appear in the papers cited in Ref. [37].

[42] The first and second questionnaires included three questions: A question on ideal gas expansion (see Appendix) taken from B.R. Bucy, J.R. Thompson, and D. B. Mountcastle, What is entropy? Advanced undergraduate performance comparing ideal gas processes, AIP Conf. Proc. 818, 81 (2006), the thermal equilibration problem from Ref. [31] shown in Table I, and the main question on phase separation. The third questionnaire encompassed only two questions: the problem on phase separation and a problem on self-assembly of micelles. We consider the problems in the three questionnaires to be isomorphic, because the physical principles required to solve the problems are the same and the procedure in which they are applied to various subproblems is the same even though the context to which these principles apply varies.

[43] This dispersion is indeed similar to the two-component mixture since the protein component only acts to stabilize the droplets that do not change their structure during the phase separation process. The phase separation is therefore between two phases with different concentrations of droplets whose sizes and shapes are the same.

[44] M.S. Sabella and E. F. Redish Knowledge organization and activation in physics problem solving, Am. J. Phys. 75, 1017 (2007).

[45] A. A. diSessa, Towards an epistemology of physics, Cognit. Instr. 10, 105 (1993).

[46] E. C. Sayre and M. C. Wittmann, Plasticity of intermediate mechanics students' coordinate system choice, Phys. Rev. ST Phys. Educ. Res. 4, 020105 (2008).

[47] The first questionnaire differs from the other two questionnaires in two aspects. (1) In order to match the vocabulary used in stage $A$, where we used the term disorder rather than a quantitative definition of entropy, the problem statement did not use of the term "entropy," but rather the terms "disorder" and "number of possible configurations." (2) Section C of the problem was replaced with a question that asked the students to determine the physical law that drives the process. This focus was a better fit to the initial stage of the program since it directed students towards path II, which was the focus of instruction in this stage. Yet as it did not serve to probe how students invoke other paths, we later replaced it with the aforementioned question.

[48] We did not describe here more complicated solution paths for part A. which can also be used to solve the entire problem. For example, one may use the idea that the change in free energy must be negative $\Delta F=$ $\Delta U-T \Delta S<0$ and deduce from the fact that the entropy decreases $\Delta S<0$ that the internal energy must decrease as well $\Delta U<0$.

[49] M.T.H. Chi, Quantifying qualitative analyses of verbal data: A practical guide, J. Learn. Sci. 6, 271 (1997).

[50] E. C. Sayre, S. V. Franklin, S. Dymek, J. Clark, and Y. Sun, Learning, retention, and forgetting of Newton's third law throughout university physics, Phys. Rev. ST Phys. Educ. Res. 8, 010116 (2012).

[51] R. Siegler, U-shaped interest in U-shaped developmentAnd what it means, J. Cognit. Dev. 5, 1 (2004). 
[52] E.F. Redish and D. Hammer, Reinventing college physics for biologists: Explicating an epistemological curriculum, Am. J. Phys. 77, 629 (2009).

[53] Ruben's answer to part C of the first questionnaire, which asks for the law that governs the process, reveals that even though he did not relate a change in potential energy to the interparticle interactions, he is aware of their existence and importance in driving the process: "The law (driving the process) is that molecules will always be attracted to other molecules with which they can form chemical bonds."

[54] One possible explanation is that Ruben did not recollect that the application of the first law of thermodynamics requires consideration of both work and heat transfer. Another possibility is that Ruben believed that once the temperature is constant there is no need to consider heat transfer between the system and its surrounding. The belief that no heat transfer occurs in isothermal processes is mentioned in Ref. [17].

[55] In fact, the effect of the change in potential energy due to the aggregation is much more dramatic than the change in potential energy due to gravitation (which is negligible in systems at the nanometer or micron scale); therefore, the change in gravitational energy has only a minor effect on the decrease in internal energy.

[56] Indeed, in this early stage most students with physics background considered the potential energy of the system, in contrast to many of the chemistry students who had not referred to the potential energy.

[57] One may claim that his justification was not complete as he did not refer to the average kinetic energy component of the internal energy in his reasoning. In fact, this is a reflection of the instruction, which emphasized the contribution of interactions to changes in internal energy. In most analyses of equilibrium soft matter systems, the average kinetic energy is a constant (independent of particle configurations) at fixed temperature and only internal energy associated with the potential interactions needs to be considered when determining the structure and thermodynamic state of the system.

[58] When considering the entire group, $27 \%$ of the students still did not mention the contribution of potential energy of interactions to the internal energy. The rest of the students acknowledged this contribution, yet 55\% incorrectly asserted that energy increases due to interparticle attractions.

[59] Ruben expressed the notion that processes are driven by a minimization of energy again, later in the course, while working on a troubleshooting activity, in which the question regarding milk fat was presented with an anonymous peer erroneous answer (namely, "The energy of the system is the same in the initial and the final state since the average kinetic energy of the system remains the same.'), In response, Ruben and his partner suggested an alternative answer, "The energy of the system decreases naturally in every spontaneous process (every system wants to be at minimum energy). In this system a spontaneous process occurred in which the milk got separated into two different phases (microscopic globules of fat and water). And since phase separation has occurred, there was a decrease in the system energy."

[60] This assertion is supported through his response regarding the change in entropy during the ideal gas expansion:
"The disorder has increased because the number of particles hasn't changed but the volume has increased, ... Disorder depends also on temperature."

[61] In a closed system that undergoes isothermal changes, the distribution of thermal energy (including the average kinetic energy per particle) does not change.

[62] This entropy increase can arise either from an increase in the energy distribution entropy (appropriate to surroundings that are homogeneous fluids or gases) or from an increase in the spatial configuration entropy (in surroundings that contain, for example, a low-concentration phase of colloidal particles). The description and the associated learning related to the detailed nature of the "surroundings" are outside the scope of this paper.

[63] Temperature is an intensive thermodynamic property (average kinetic energy per particle) that does not depend on the system size in the thermodynamic limit of an infinitely large system. In that limit, transferring a small amount of energy from the system increases the entropy of the surroundings by an amount proportional to the size of the system which is much smaller than that of the surroundings. In such cases, the temperature of the surroundings which satisfies $1 / T=\Delta S / \Delta E$ does not change, although its (extensive) entropy and energy have changed due to the heat transfer.

[64] This assertion is supported by triangulation with Ruben's response to the thermal equilibration problem (Table I) in which he claimed that the entropy of the air in the room and the object does not change.

[65] He answered that the entropy of the object and the air in the room must increase in thermal equilibration.

[66] About $46 \%$ of the students made similar, incorrect statements that allowed for violations of the second law in systems in which system entropy decrease.

[67] T. Levy-Nahum, R. Mamlok-Naaman, A Hofstein, and K.S. Taber, Teaching and learning the concept of chemical bonding, Stud. Sci. Educ. 46, 179 (2010).

[68] K.C. Lee, How to teach statistical thermal physics in an introductory physics course, Am. J. Phys. 69, 68 (2001).

[69] Inspired by the chapter "Egyptian Ink," P. G. de Gennes and J. Badoz, Fragile Objects: Soft Matter, Hard Science, and the Thrill of Discovery (Springer-Verlag, New York, 1996), pp. 20-39.

[70] D. F. Styer, Insight into entropy, Am. J. Phys. 68, 1090 (2000).

[71] The complexity of discussing energy levels (e.g., using the Einstein solid model, see, for example, Ref. [8]) was avoided. Instead, an expression for the entropy was derived by distributing a certain amount of equal sized ("digitized") energy quanta among the particles in the spirit of Ref. [7].

[72] See Chang's textbook in Ref. [26], pp. 735-737.

[73] In order to calculate the average interaction energy we use several simplifications: (1) Only nearest neighbors contribute since intermolecular forces are short ranged. (2) Interaction energy between neighboring particles is taken to be a constant (depending only on the particle type) since the particles are located on a lattice. (3) Mean-field approximation: the nearest neighbors' composition corresponds to the average particle composition in the entire system. 